\title{
Redefined intuitionistic fuzzy bi-ideals of ordered semigroups
}

\author{
Asghar Khan ${ }^{*}$, Bijan Davvaz², Nor Haniza Sarmin ${ }^{3}$ and Hidayatullah Khan ${ }^{3}$
}

"Correspondence:

azhar4set@yahoo.com

${ }^{1}$ Department of Mathematics,

Abdul Wali Khan University, Mardan,

Khyber Pakhtoon Khwa, Pakistan

Full list of author information is

available at the end of the article

\begin{abstract}
In this article, we try to obtain a more general form than $(\in, \in \vee \mathrm{q})$-intuitionistic fuzzy bi-ideals in ordered semigroups. The notion of an $\left(\in, \in \vee q_{k}\right)$-intuitionistic fuzzy bi-ideal is introduced, and several properties are investigated. Characterizations of an $\left(\in, \in \vee \mathbf{q}_{k}\right)$-intuitionistic fuzzy bi-ideal are established. A condition for an $\left(\in, \in \vee \mathrm{q}_{k}\right)$-intuitionistic fuzzy bi-ideal to be an intuitionistic fuzzy bi-ideal is provided. It is shown that every $(\epsilon, \epsilon)$-intuitionistic fuzzy bi-ideal is an $(\epsilon, \in \vee \mathrm{q})$-intuitionistic fuzzy bi-ideal, and every $(\in, \in \vee \mathrm{q})$-intuitionistic fuzzy bi-ideal is an $\left(\in, \in \vee \mathrm{q}_{k}\right)$-intuitionistic fuzzy bi-ideal but the converse is not true. The important achievement of the study with an $\left(\in, \in \vee q_{k}\right)$-intuitionistic fuzzy bi-ideal is that the notion of an $(\in, \in \vee \mathrm{q})$-intuitionistic fuzzy bi-ideal is a special case of an $\left(\in, \in \vee \mathrm{q}_{k}\right)$-intuitionistic fuzzy bi-ideal, and, thus, several results in the paper (Jun et al. in Bi-ideals of ordered semigroups based on the intuitionistic fuzzy points (submitted)) are the corollaries of our results obtained in this paper.
\end{abstract}

Keywords: intuitionistic fuzzy bi-ideal; $(\in, \in \vee \mathrm{q})$-intuitionistic fuzzy bi-ideal; $\left(\in, \in \vee \mathrm{q}_{k}\right)$-intuitionistic fuzzy bi-ideal

\section{Introduction}

In mathematics, an ordered semigroup is a semigroup together with a partial order that is compatible with the semigroup operation. Ordered semigroups have many applications in the theory of sequential machines, formal languages, computer arithmetics, design of fast adders and error-correcting codes. The concept of a fuzzy filter in ordered semigroups was first introduced by Kehayopulu and Tsingelis in [1], where some basic properties of fuzzy filters and prime fuzzy ideals were discussed. A theory of fuzzy generalized sets on ordered semigroups can be developed. Mordeson et al. in [2] presented an up-to-date account of fuzzy sub-semigroups and fuzzy ideals of a semigroup. Murali [3] proposed the definition of a fuzzy point belonging to a fuzzy subset under a natural equivalence on fuzzy subset. The idea of quasi-coincidence of a fuzzy point with a fuzzy set played a vital role in generating different types of fuzzy subgroups. Bhakat and Das $[4,5]$ gave the concepts of $(\alpha, \beta)$-fuzzy subgroups by using the 'belong to' $(\epsilon)$ relation and 'quasi-coincident with' (q) relation between a fuzzy point and a fuzzy subgroup, and introduced the concept of $(\in, \in \vee \mathrm{q})$-fuzzy subgroup. In [6], Davvaz started the generalized fuzzification in algebra. In [7], Jun et al. initiated the study of $(\alpha, \beta)$-fuzzy bi-ideals of an ordered semigroup. In [8], Davvaz and Khan studied $(\epsilon, \in \vee \mathrm{q})$-fuzzy generalized bi-ideals of an ordered semigroup. Shabir et al. [9] studied characterization of regular semigroups by $(\alpha, \beta)$-fuzzy ideals. Jun

C 2013 Khan et al.: licensee Springer. This is an Open Access article distributed under the terms of the Creative Commons Attribution License (http://creativecommons.org/licenses/by/2.0), which permits unrestricted use, distribution, and reproduction in any medium, provided the original work is properly cited. 
et al. [10] discussed a generalization of $(\in, \in \vee \mathrm{q})$-fuzzy ideals of a $B C K / B C I$-algebra. Using the idea of a quasi-coincidence of a fuzzy point with a fuzzy set, Jun et al. [11] introduced the concept of $(\alpha, \beta)$-intuitionistic fuzzy bi-ideals in an ordered semigroup. They introduced a new sort of intuitionistic fuzzy bi-ideals, called $(\alpha, \beta)$-intuitionistic fuzzy biideals, and studied $(\in, \in \vee \mathrm{q})$-intuitionistic fuzzy bi-ideals.

In this paper, we try to have more general form of an $(\in, \in \vee \mathrm{q})$-intuitionistic bi-ideal of an ordered semigroup. We introduce the notion of an $\left(\in, \in \vee \mathrm{q}_{k}\right)$-intuitionistic bi-ideal of an ordered semigroup, and give examples which are $\left(\in, \in \vee \mathrm{q}_{k}\right)$-intuitionistic fuzzy bi-ideals but not $(\in, \in \vee \mathrm{q})$-intuitionistic fuzzy bi-ideals. We discuss characterizations of $\left(\in, \in \vee \mathrm{q}_{k}\right)$-intuitionistic fuzzy bi-ideals in ordered semigroups. We provide a condition for an $\left(\in, \in \vee \mathrm{q}_{k}\right)$-intuitionistic fuzzy bi-ideal to be an intuitionistic fuzzy bi-ideal. The important achievement of the study with an $\left(\in, \in \vee \mathrm{q}_{k}\right)$-intuitionistic fuzzy bi-ideal is that the notion of an $(\in, \in \vee \mathrm{q})$-intuitionistic fuzzy bi-ideal is a special case of an $\left(\in, \in \vee \mathrm{q}_{k}\right)$ intuitionistic fuzzy bi-ideal, and, thus, several results in the paper [11] are the corollaries of our results obtained in this paper.

\section{Basic definitions and preliminary results}

By an ordered semigroup (or po-semigroup) we mean a structure $(S, \cdot, \leq)$, in which the following are satisfied:

(OS1) $(S, \cdot)$ is a semigroup,

(OS2) $(S, \leq)$ is a poset,

(OS3) $(\forall x, a, b \in S)(a \leq b \Rightarrow a \cdot x \leq b \cdot x, x \cdot a \leq x \cdot b)$.

In what follows, $x \cdot y$ is simply denoted by $x y$ for all $x, y \in S$.

A nonempty subset $A$ of an ordered semigroup $S$ is called a subsemigroup of $S$ if $A^{2} \subseteq A$. A non-empty subset $A$ of an ordered semigroup $S$ is called a bi-ideal of $S$ if it satisfies

(b1) $(\forall b \in S)(\forall b \in A)(a \leq b \Rightarrow b \in A)$,

(b2) $(\forall a, b \in S)(a, b \in A \Rightarrow a b \in A)$,

(b3) $A S A \subseteq A$.

An intuitionistic fuzzy set (briefly IFS) $A$ in a non-empty set $X$ is an object having the form $A=\left\{\left\langle x, \mu_{A}(x), \gamma_{A}(x)\right\rangle \mid x \in X\right\}$, where the function $\mu_{A}: X \longrightarrow[0,1]$ and $\gamma_{A}: X \longrightarrow[0,1]$ denote the degree of membership (namely, $\mu_{A}(x)$ ) and the degree of non-membership (namely, $\gamma_{A}(x)$ ) for each element $x \in X$ to the set $A$, respectively, and $0 \leq \mu_{A}(x)+\gamma_{A}(x) \leq 1$ for all $x \in X$. For the sake of simplicity, we shall use the symbol $A=\left\langle x, \mu_{A}, \gamma_{A}\right\rangle$ for the intuitionistic fuzzy set $A=\left\{\left\langle x, \mu_{A}(x), \gamma_{A}(x)\right\rangle \mid x \in X\right\}$.

Let $(S, \cdot, \leq)$ be an ordered semigroup and $A=\left\langle x, \mu_{A}, \gamma_{A}\right\rangle$ be an IFS of $S$. Then $A=$ $\left\langle x, \mu_{A}, \gamma_{A}\right\rangle$ is called an intuitionistic fuzzy subsemigroup of $S$ [11] if

$$
(\forall x, y \in S) \quad\left(\mu_{A}(x y) \geq \min \left\{\mu_{A}(x), \mu_{A}(y)\right\} \text { and } \gamma_{A}(x y) \leq \max \left\{\gamma_{A}(x), \gamma_{A}(y)\right\}\right) .
$$

Let $(S, \cdot, \leq)$ be an ordered semigroup and $A=\left\langle x, \mu_{A}, \gamma_{A}\right\rangle$ be an intuitionistic fuzzy subsemigroup of $S$. Then $A=\left\langle x, \mu_{A}, \gamma_{A}\right\rangle$ is called an intuitionistic fuzzy bi-ideal of $S$ [11] if

(b4) $(\forall x, y \in S)\left(x \leq y \Longrightarrow \mu_{A}(x) \geq \mu_{A}(y)\right.$ and $\left.\gamma_{A}(x) \leq \gamma_{A}(y)\right)$,

(b5) $(\forall x, y \in S)\left(\mu_{A}(x y) \geq \min \left\{\mu_{A}(x), \mu_{A}(y)\right\}\right.$ and $\left.\gamma_{A}(x y) \leq \max \left\{\gamma_{A}(x), \gamma_{A}(y)\right\}\right)$,

(b6) $(\forall x, y, z \in S)\left(\mu_{A}(x y z) \geq \min \left\{\mu_{A}(x), \mu_{A}(z)\right\}\right.$ and $\left.\gamma_{A}(x y z) \leq \max \left\{\gamma_{A}(x), \gamma_{A}(z)\right\}\right)$.

Let $A=\left\langle x, \mu_{A}, \gamma_{A}\right\rangle$ be an IFS of $S$ and $\alpha \in(0,1]$ and $\beta \in[0,1)$. Then the sets

$$
U\left(\mu_{A} ; \alpha\right)=\left\{x \in S \mid \mu_{A}(x) \geq \alpha\right\} \quad \text { and } \quad L\left(\gamma_{A} ; \beta\right)=\left\{x \in S \mid \gamma_{A}(x) \leq \beta\right\}
$$


are called $\mu_{A}$-level and $\gamma_{A}$-level cuts of the intuitionistic fuzzy set $A=\left\langle x, \mu_{A}, \gamma_{A}\right\rangle$, respectively. For an IFS $A=\left\langle x, \mu_{A}, \gamma_{A}\right\rangle$ and $\alpha \in(0,1], \beta \in[0,1)$, we define the $\left(\mu_{A}, \gamma_{A}\right)$-level cut as follows

$$
C_{(\alpha, \beta)}(A)=\left\{x \in S \mid \mu_{A}(x) \geq \alpha \text { and } \gamma_{A}(x) \leq \beta\right\}
$$

Clearly, $C_{(\alpha, \beta)}(A)=U\left(\mu_{A} ; \alpha\right) \cap L\left(\gamma_{A} ; \beta\right)$.

Let $x$ be a point of a non-empty set $X$. If $\alpha \in(0,1]$ and $\beta \in[0,1)$ are two real numbers such that $0 \leq \alpha+\beta \leq 1$, then the IFS of the form

$$
\langle x ;(\alpha, \beta)\rangle=\left\langle x ; x_{\alpha}, 1-x_{1-\beta}\right\rangle
$$

is called an intuitionistic fuzzy point (IFP for short) in $X$, where $\alpha$ (resp. $\beta$ ) is the degree of membership (resp. non-membership) of $\langle x ;(\alpha, \beta)\rangle$ and $x \in X$ is the support of $\langle x ;(\alpha, \beta)\rangle$.

Consider an IFP $\langle x ;(\alpha, \beta)\rangle$ in $S$, an IFS $A=\left\langle x, \mu_{A}, \gamma_{A}\right\rangle$ and $\alpha \in\{\in, \mathrm{q}, \in \vee \mathrm{q}\}$, we define $\langle x ;(\alpha, \beta)\rangle \alpha A$ as follows

(b7) $\langle x ;(\alpha, \beta)\rangle \in A$ (resp. $\langle x ;(\alpha, \beta)\rangle \mathrm{q} A$ ) means that $\mu_{A}(x) \geq \alpha$ and $\gamma_{A}(x) \leq \beta$ (resp. $\mu_{A}(x)+\alpha>1$ and $\left.\gamma_{A}(x)+\beta<1\right)$, and in this case, we say that $\langle x ;(\alpha, \beta)\rangle$ belongs to (resp. quasi-coincident with) an IFS $A=\left\langle x, \mu_{A}, \gamma_{A}\right\rangle$.

(b8) $\langle x ;(\alpha, \beta)\rangle \in \vee \mathrm{q} A$ (resp. $\langle x ;(\alpha, \beta)\rangle \in \wedge \mathrm{q} A$ ) means that $\langle x ;(\alpha, \beta)\rangle \in A$ or $\langle x ;(\alpha, \beta)\rangle \mathrm{q} A$ (resp. $\langle x ;(\alpha, \beta)\rangle \in A$ and $\langle x ;(\alpha, \beta)\rangle \mathrm{q} A)$.

By $\langle x ;(\alpha, \beta)\rangle \bar{\alpha} A$, we mean that $\langle x ;(\alpha, \beta)\rangle \alpha A$ does not hold.

\section{$3\left(\epsilon, \in \vee q_{k}\right)$-Intuitionistic fuzzy bi-ideals}

Let $k$ denote an arbitrary element of $[0,1)$ unless specified otherwise. For an IFP $\langle x ;(\alpha, \beta)\rangle$ and an IFS $A=\left\langle x, \mu_{A}, \gamma_{A}\right\rangle$ of $X$, we say that

(c1) $\langle x ;(\alpha, \beta)\rangle \mathrm{q}_{k} A$ if $\mu_{A}(x)+k+\alpha>1$ and $\gamma_{A}(x)+k+\beta<1$.

(c2) $\langle x ;(\alpha, \beta)\rangle \in \vee \mathrm{q}_{k} A$ if $\langle x ;(\alpha, \beta)\rangle \in A$ or $\langle x ;(\alpha, \beta)\rangle \mathrm{q}_{k} A$.

(c3) $\langle x ;(\alpha, \beta)\rangle \bar{\alpha} A$ if $\langle x ;(\alpha, \beta)\rangle \alpha A$ does not hold for $\alpha \in\left\{\mathrm{q}_{k}, \in \vee \mathrm{q}_{k}\right\}$.

Theorem 3.1 Let $A=\left\langle x, \mu_{A}, \gamma_{A}\right\rangle$ be an IFS of an ordered semigroup $S$. Then the following are equivalent

(1) $\left(\forall \alpha \in\left(\frac{1-k}{2}, 1\right]\right)\left(\forall \beta \in\left[0, \frac{1-k}{2}\right)\right)\left(C_{(\alpha, \beta)}(A) \neq \emptyset \Longrightarrow C_{(\alpha, \beta)}(A)\right.$ is a bi-ideal of $\left.S\right)$.

(2) $A=\left\langle x, \mu_{A}, \gamma_{A}\right\rangle$ satisfies the following assertions

$$
\begin{aligned}
& \left(\begin{array}{l}
x \leq y \Longrightarrow \mu_{A}(y) \leq \max \left\{\mu_{A}(x), \frac{1-k}{2}\right\} \\
\text { and } \gamma_{A}(y) \geq \min \left\{\gamma_{A}(x), \frac{1-k}{2}\right\}
\end{array}\right), \\
& \left(\begin{array}{l}
\min \left\{\mu_{A}(x), \mu_{A}(y)\right\} \leq \max \left\{\mu_{A}(x y), \frac{1-k}{2}\right\} \\
\text { and } \max \left\{\gamma_{A}(x), \gamma_{A}(y)\right\} \geq \min \left\{\gamma_{A}(x y), \frac{1-k}{2}\right\}
\end{array}\right), \\
& \left(\begin{array}{l}
\min \left\{\mu_{A}(x), \mu_{A}(z)\right\} \leq \max \left\{\mu_{A}(x y z), \frac{1-k}{2}\right\} \text { and } \\
\max \left\{\gamma_{A}(x), \gamma_{A}(z)\right\} \geq \min \left\{\gamma_{A}(x y z), \frac{1-k}{2}\right\}
\end{array}\right),
\end{aligned}
$$

for all $x, y, z \in S$.

Proof Assume that $C_{(\alpha, \beta)}(A)$ is a bi-ideal of $S$ for all $\alpha \in\left(\frac{1-k}{2}, 1\right]$ and $\beta \in\left[0, \frac{1-k}{2}\right)$ with $C_{(\alpha, \beta)}(A) \neq \emptyset$. If there exist $a, b \in S$ such that condition (2.1) is not valid, that is, there exist $a, b \in S$ with $a \leq b$ and $\mu_{A}(b)>\max \left\{\mu_{A}(a), \frac{1-k}{2}\right\}, \gamma_{A}(b)<\min \left\{\gamma_{A}(a), \frac{1-k}{2}\right\}$. Then $\mu_{A}(b) \in$ 
$\left(\frac{1-k}{2}, 1\right], \gamma_{A}(b) \in\left[0, \frac{1-k}{2}\right)$ and $b \in C_{\left(\mu_{A}(b), \gamma_{A}(b)\right)}(A)$. But $\mu_{A}(a)<\mu_{A}(b)$ and $\gamma_{A}(a)>\gamma_{A}(b)$ imply that $a \notin C_{\left(\mu_{A}(b), \gamma_{A}(b)\right)}(A)$. This is not possible. Hence (2.1) is valid. Suppose that (2.2) is false, that is,

$$
s:=\min \left\{\mu_{A}(a), \mu_{A}(c)\right\}>\max \left\{\mu_{A}(a c), \frac{1-k}{2}\right\}
$$

and

$$
t:=\max \left\{\gamma_{A}(a), \gamma_{A}(c)\right\}<\min \left\{\gamma_{A}(a c), \frac{1-k}{2}\right\}
$$

for some $a, c \in S$. Then $s \in\left(\frac{1-k}{2}, 1\right], t \in\left[0, \frac{1-k}{2}\right)$ and $a, c \in C_{(s, t)}(A)$. But $a c \notin C_{(s, t)}(A)$, since $\mu_{A}(a c)<s$ and $\gamma_{A}(a c)>t$. This is not possible, and so (2.2) is valid. If there exist $a, b, c \in S$ such that (2.3) is not valid, that is,

$$
s_{0}:=\min \left\{\mu_{A}(a), \mu_{A}(b)\right\}>\max \left\{\mu_{A}(a c b), \frac{1-k}{2}\right\}
$$

and

$$
t_{0}:=\max \left\{\gamma_{A}(a), \gamma_{A}(b)\right\}<\min \left\{\gamma_{A}(a c b), \frac{1-k}{2}\right\}
$$

Then $s_{0} \in\left(\frac{1-k}{2}, 1\right], t_{0} \in\left[0, \frac{1-k}{2}\right)$ and $a, b \in C_{\left(s_{0}, t_{0}\right)}(A)$. But $a c b \notin C_{\left(s_{0}, t_{0}\right)}(A)$, since $\mu_{A}(a c b)<$ $s_{0}$ and $\gamma_{A}(a c b)>t_{0}$. This is a contradiction, and hence (2.3) is valid.

Conversely, assume that $A=\left\langle x, \mu_{A}, \gamma_{A}\right\rangle$ satisfies the three conditions (2.1), (2.2) and (2.3). Suppose that $C_{(\alpha, \beta)}(A) \neq \emptyset$ for all $\alpha \in\left(\frac{1-k}{2}, 1\right]$, and $\beta \in\left[0, \frac{1-k}{2}\right)$. Let $x, y \in S$ be such that $x \leq y$ and $y \in C_{(\alpha, \beta)}(A)$. Then $\mu_{A}(y) \geq \alpha$ and $\gamma_{A}(y) \leq \beta$. Using (2.1), we have $\max \left\{\mu_{A}(x), \frac{1-k}{2}\right\} \geq$ $\mu_{A}(y) \geq \alpha>\frac{1-k}{2}$ and $\min \left\{\gamma_{A}(x), \frac{1-k}{2}\right\} \leq \gamma_{A}(y) \leq \beta<\frac{1-k}{2}$. Hence $\mu_{A}(x) \geq \alpha$ and $\gamma_{A}(x) \leq \beta$, i.e., $x \in C_{(\alpha, \beta)}(A)$. If $x, y \in C_{(\alpha, \beta)}(A)$, then $\mu_{A}(x) \geq \alpha, \gamma_{A}(x) \leq \beta$ and $\mu_{A}(y) \geq \alpha, \gamma_{A}(y) \leq \beta$. By using (2.2), we have

$$
\max \left\{\mu_{A}(x y), \frac{1-k}{2}\right\} \geq \min \left\{\mu_{A}(x), \mu_{A}(y)\right\} \geq \alpha>\frac{1-k}{2}
$$

and

$$
\min \left\{\gamma_{A}(x y), \frac{1-k}{2}\right\} \leq \max \left\{\gamma_{A}(x), \gamma_{A}(y)\right\} \leq \beta<\frac{1-k}{2},
$$

so that $\mu_{A}(x y) \geq \alpha$ and $\gamma_{A}(x y) \leq \beta$, i.e., $x y \in C_{(\alpha, \beta)}(A)$. Finally, if $x, z \in C_{(\alpha, \beta)}(A)$ and $y \in S$, then $\mu_{A}(x) \geq \alpha, \gamma_{A}(x) \leq \beta$ and $\mu_{A}(z) \geq \alpha, \gamma_{A}(z) \leq \beta$. By using (2.3), we have

$$
\max \left\{\mu_{A}(x y z), \frac{1-k}{2}\right\} \geq \min \left\{\mu_{A}(x), \mu_{A}(z)\right\} \geq \alpha>\frac{1-k}{2}
$$

and

$$
\min \left\{\gamma_{A}(x y z), \frac{1-k}{2}\right\} \leq \max \left\{\gamma_{A}(x), \gamma_{A}(z)\right\} \leq \beta<\frac{1-k}{2},
$$


Table $1 *$-multiplication table for $S$

\begin{tabular}{l|l|l|l|l|l}
$*$ & $a$ & $b$ & $c$ & $d$ & $e$ \\
\hline$a$ & $a$ & $d$ & $a$ & $d$ & $d$ \\
\hline$b$ & $a$ & $b$ & $a$ & $d$ & $d$ \\
\hline$c$ & $a$ & $d$ & $c$ & $d$ & $e$ \\
\hline$d$ & $a$ & $d$ & $a$ & $d$ & $d$ \\
\hline$e$ & $a$ & $d$ & $c$ & $d$ & $e$
\end{tabular}

so that $\mu_{A}(x y z) \geq \alpha$ and $\gamma_{A}(x y z) \leq \beta$, i.e., $x y z \in C_{(\alpha, \beta)}(A)$. Therefore, $C_{(\alpha, \beta)}(A)$ is a bi-ideal of $S$.

If we take $k=0$ in Theorem 3.1, then we have the following corollary.

Corollary 3.2 [11, Theorem 3.1] Let $A=\left\langle x, \mu_{A}, \gamma_{A}\right\rangle$ be an IFS of S. Then the following assertions are equivalent

(1) $(\forall \alpha \in(0.5,1])(\forall \beta \in[0,0.5))\left(C_{(\alpha, \beta)}(A) \neq \emptyset \Longrightarrow C_{(\alpha, \beta)}(A)\right.$ is a bi-ideal of $\left.S\right)$.

(2) $A=\left\langle x, \mu_{A}, \gamma_{A}\right\rangle$ satisfies the following conditions

$$
\begin{aligned}
& \left(\begin{array}{l}
x \leq y \Longrightarrow \mu_{A}(y) \leq \max \left\{\mu_{A}(x), 0.5\right\} \\
\text { and } \gamma_{A}(y) \geq \min \left\{\gamma_{A}(x), 0.5\right\}
\end{array}\right), \\
& \left(\begin{array}{l}
\min \left\{\mu_{A}(x), \mu_{A}(y)\right\} \leq \max \left\{\mu_{A}(x y), 0.5\right\} \\
\text { and } \max \left\{\gamma_{A}(x), \gamma_{A}(y)\right\} \geq \min \left\{\gamma_{A}(x y), 0.5\right\}
\end{array}\right), \\
& \left(\begin{array}{l}
\min \left\{\mu_{A}(x), \mu_{A}(z)\right\} \leq \max \left\{\mu_{A}(x y z), 0.5\right\} \\
\text { and } \max \left\{\gamma_{A}(x), \gamma_{A}(z)\right\} \geq \min \left\{\gamma_{A}(x y z), 0.5\right\}
\end{array}\right),
\end{aligned}
$$

for all $x, y, z \in S$.

Definition 3.3 An IFS $A=\left\langle x, \mu_{A}, \gamma_{A}\right\rangle$ in $S$ is called an $\left(\epsilon, \in \vee \mathrm{q}_{k}\right)$-intuitionistic fuzzy biideal of $S$ if for all $x, y, z \in S, t, t_{1}, t_{2} \in(0,1]$ and $s, s_{1}, s_{2} \in[0,1)$ it satisfies the following conditions

(q1) $\left(x \leq y,\langle y ;(t, s)\rangle \in A \Longrightarrow\langle x ;(t, s)\rangle \in \vee \mathrm{q}_{k} A\right)$,

(q2) $\left(\left\langle x ;\left(t_{1}, s_{1}\right)\right\rangle \in A\right.$ and $\left.\left\langle y ;\left(t_{2}, s_{2}\right)\right\rangle \in A \Longrightarrow\left\langle x y ; \min \left\{t_{1}, t_{2}\right\}, \max \left\{s_{1}, s_{2}\right\}\right\rangle \in \vee \mathrm{q}_{k} A\right)$,

(q3) $\left(\left\langle x ;\left(t_{1}, s_{1}\right)\right\rangle \in A\right.$ and $\left.\left\langle z ;\left(t_{2}, s_{2}\right)\right\rangle \in A \Longrightarrow\left\langle x y z ; \min \left\{t_{1}, t_{2}\right\}, \max \left\{s_{1}, s_{2}\right\}\right\rangle \in \vee \mathrm{q}_{k} A\right)$.

An $\left(\in, \in \vee \mathrm{q}_{k}\right)$-intuitionistic fuzzy bi-ideal of $S$ with $k=0$ is an $(\in, \in \vee \mathrm{q})$-intuitionistic fuzzy bi-ideal of $S$.

Example 3.4 Consider the set $S=\{a, b, c, d, e\}$ with the order relation $a \leq c \leq e, a \leq d \leq e$, $b \leq d$ and $b \leq e$ and $*$-multiplication table (see Table 1 above).

(1) Define an IFS $A=\left\langle x, \mu_{A}, \gamma_{A}\right\rangle$ by

$$
\mu_{A}: S \rightarrow[0,1] \mid \mu_{A}(x)= \begin{cases}0.40 & \text { if } x=a, \\ 0.35 & \text { if } x=b, \\ 0.30 & \text { if } x=c \\ 0.50 & \text { if } x=d, \\ 0.20 & \text { if } x=e\end{cases}
$$


and

$$
\gamma_{A}: S \rightarrow[0,1] \mid \gamma_{A}(x)= \begin{cases}0.40 & \text { if } x=a, \\ 0.30 & \text { if } x=b, \\ 0.50 & \text { if } x=c, \\ 0.40 & \text { if } x=d, \\ 0.80 & \text { if } x=e .\end{cases}
$$

Then $A=\left\langle x, \mu_{A}, \gamma_{A}\right\rangle$ is an $\left(\in, \in \vee \mathrm{q}_{0.4}\right)$-intuitionistic fuzzy bi-ideal of $S$.

(2) Let $A=\left\langle x, \mu_{A}, \gamma_{A}\right\rangle$ be an intuitionistic fuzzy set given by

$$
\mu_{A}: S \rightarrow[0,1] \mid \mu_{A}(x)= \begin{cases}0.80 & \text { if } x=a, \\ 0.60 & \text { if } x=b, e, \\ 0.30 & \text { if } x=c, \\ 0.50 & \text { if } x=d\end{cases}
$$

and

$$
\gamma_{A}: S \rightarrow[0,1] \mid \gamma_{A}(x)= \begin{cases}0.20 & \text { if } x=a, \\ 0.30 & \text { if } x=b, e, \\ 0.60 & \text { if } x=c, \\ 0.50 & \text { if } x=d .\end{cases}
$$

Then $A=\left\langle x, \mu_{A}, \gamma_{A}\right\rangle$ is an $\left(\in, \in \vee \mathrm{q}_{0.04}\right)$-intuitionistic fuzzy bi-ideal of $S$.

Theorem 3.5 An IFS $A=\left\langle x, \mu_{A}, \gamma_{A}\right\rangle$ of $S$ is an $\left(\in, \in \vee \mathrm{q}_{k}\right)$-intuitionistic fuzzy bi-ideal of $S$ if and only if it satisfies the following conditions
(1) $\left(\begin{array}{l}x \leq y \Longrightarrow \mu_{A}(x) \geq \min \left\{\mu_{A}(y), \frac{1-k}{2}\right\} \\ \text { and } \gamma_{A}(x) \leq \max \left\{\gamma_{A}(y), \frac{1-k}{2}\right\}\end{array}\right)$,
(2) $\left(\begin{array}{l}\mu_{A}(x y) \geq \min \left\{\mu_{A}(x), \mu_{A}(y), \frac{1-k}{2}\right\} \\ \text { and } \gamma_{A}(x y) \leq \max \left\{\gamma_{A}(x), \gamma_{A}(y), \frac{1-k}{2}\right\}\end{array}\right)$,
(3) $\left(\begin{array}{l}\mu_{A}(x y z) \geq \min \left\{\mu_{A}(x), \mu_{A}(z), \frac{1-k}{2}\right\} \\ \text { and } \gamma_{A}(x y z) \leq \max \left\{\gamma_{A}(x), \gamma_{A}(z), \frac{1-k}{2}\right\}\end{array}\right)$.

Proof Suppose that $A=\left\langle x, \mu_{A}, \gamma_{A}\right\rangle$ is an $\left(\in, \in \vee \mathrm{q}_{k}\right)$-intuitionistic fuzzy bi-ideal of $S$. Let $x, y \in S$ be such that $x \leq y$. Assume that $\mu_{A}(y) \leq \frac{1-k}{2}$ and $\gamma_{A}(y) \geq \frac{1-k}{2}$. If $\mu_{A}(x)<\mu_{A}(y)$ and $\gamma_{A}(x)>\gamma_{A}(y)$, then $\mu_{A}(x)<t \leq \mu_{A}(y)$ and $\gamma_{A}(x)>s \geq \gamma_{A}(y)$ for some $t \in\left(0, \frac{1-k}{2}\right)$ and $s \in\left(\frac{1-k}{2}, 1\right)$. It follows $\langle y ;(t, s)\rangle \in A$, but $\langle x ;(t, s)\rangle \bar{\epsilon}$. Since $\mu_{A}(x)+t<2 t<1-k$ and $\gamma_{A}(x)+s>2 s>1-k$, we get $\langle x ;(t, s)\rangle \overline{\mathrm{q}_{k}} A$. Therefore, $\langle x ;(t, s)\rangle \overline{\in \vee \mathrm{q}_{k}} A$, which is a contradiction. Hence $\mu_{A}(x) \geq \mu_{A}(y)$ and $\gamma_{A}(x) \leq \gamma_{A}(y)$. Now, if $\mu_{A}(y) \geq \frac{1-k}{2}$ and $\gamma_{A}(x) \leq \frac{1-k}{2}$, then $\left\langle y ;\left(\frac{1-k}{2}, \frac{1-k}{2}\right)\right\rangle \in A$, and so, $\left\langle x ;\left(\frac{1-k}{2}, \frac{1-k}{2}\right)\right\rangle \in \vee \mathrm{q}_{k} A$, which implies that $\left\langle x ;\left(\frac{1-k}{2}, \frac{1-k}{2}\right)\right\rangle \in A$ or $\left\langle x ;\left(\frac{1-k}{2}, \frac{1-k}{2}\right)\right\rangle \mathrm{q}_{k} A$, that is, $\mu_{A}(x) \geq \frac{1-k}{2}$ and $\gamma_{A}(x) \leq \frac{1-k}{2}$ or $\mu_{A}(x)+\frac{1-k}{2}>1$ and $\gamma_{A}(x)+\frac{1-k}{2}<1$. 
Hence $\mu_{A}(x) \geq \frac{1-k}{2}$ and $\gamma_{A}(x) \leq \frac{1-k}{2}$. Otherwise, $\mu_{A}(x)+\frac{1-k}{2}<\frac{1-k}{2}+\frac{1-k}{2}=1$ and $\gamma_{A}(x)+\frac{1-k}{2}>$ $\frac{1-k}{2}+\frac{1-k}{2}=1$, a contradiction. Consequently,

$$
\mu_{A}(x) \geq \min \left\{\mu_{A}(y), \frac{1-k}{2}\right\}
$$

and

$$
\gamma_{A}(x) \leq \max \left\{\gamma_{A}(y), \frac{1-k}{2}\right\}
$$

for all $x, y \in S$ with $x \leq y$. Let $x, y \in S$ be such that $\min \left\{\mu_{A}(x), \mu_{A}(y)\right\}<\frac{1-k}{2}$ and $\max \left\{\gamma_{A}(x), \gamma_{A}(y)\right\}>\frac{1-k}{2}$. We claim that $\mu_{A}(x y) \geq \min \left\{\mu_{A}(x), \mu_{A}(y)\right\}$ and $\gamma_{A}(x y) \leq$ $\max \left\{\gamma_{A}(x), \gamma_{A}(y)\right\}$. If not, then $\mu_{A}(x y)<t \leq \min \left\{\mu_{A}(x), \mu_{A}(y)\right\}$ and $\gamma_{A}(x y)>s \geq \max \left\{\gamma_{A}(x)\right.$, $\left.\gamma_{A}(y)\right\}$ for some $t \in\left(0, \frac{1-k}{2}\right)$ and $s \in\left(\frac{1-k}{2}, 1\right)$. It follows that $\langle x ;(t, s)\rangle \in A$ and $\langle y ;(t, s)\rangle \in A$, but $\langle x y ;(t, s)\rangle \bar{\epsilon} A$ and $\mu_{A}(x y)+t<2 t<1-k$ and $\gamma_{A}(x y)+s>2 s>1-k$, i.e., $\langle x y ;(t, s)\rangle \overline{\mathrm{q}_{k}} A$. This is a contradiction. Thus, $\mu_{A}(x y) \geq \min \left\{\mu_{A}(x), \mu_{A}(y)\right\}$ and $\gamma_{A}(x y) \leq \max \left\{\gamma_{A}(x), \gamma_{A}(y)\right\}$ for all $x, y \in S$ with $\min \left\{\mu_{A}(x), \mu_{A}(y)\right\}<\frac{1-k}{2}$ and $\max \left\{\gamma_{A}(x), \gamma_{A}(y)\right\}>\frac{1-k}{2}$. If $\min \left\{\mu_{A}(x), \mu_{A}(y)\right\} \geq$ $\frac{1-k}{2}$ and $\max \left\{\gamma_{A}(x), \gamma_{A}(y)\right\} \leq \frac{1-k}{2}$, then $\left\langle x ;\left(\frac{1-k}{2}, \frac{1-k}{2}\right)\right\rangle \in A$ and $\left\langle y ;\left(\frac{1-k}{2}, \frac{1-k}{2}\right)\right\rangle \in A$. Using (q2), we have

$$
\begin{aligned}
& \left\langle x y ;\left(\frac{1-k}{2}, \frac{1-k}{2}\right)\right\rangle \\
& \quad=\left\langle x y ; \min \left\{\frac{1-k}{2}, \frac{1-k}{2}\right\}, \max \left\{\frac{1-k}{2}, \frac{1-k}{2}\right\}\right\rangle \in \vee \mathrm{q}_{k} A,
\end{aligned}
$$

and so, $\mu_{A}(x y) \geq \frac{1-k}{2}$ and $\gamma_{A}(x y) \leq \frac{1-k}{2}$ or $\mu_{A}(x y)+\frac{1-k}{2}>1$ and $\gamma_{A}(x y)+\frac{1-k}{2}<1$. If $\mu_{A}(x y)<$ $\frac{1-k}{2}$ and $\gamma_{A}(x y)>\frac{1-k}{2}$, then

$$
\mu_{A}(x y)+\frac{1-k}{2}<\frac{1-k}{2}+\frac{1-k}{2}=1
$$

and

$$
\gamma_{A}(x y)+\frac{1-k}{2}>\frac{1-k}{2}+\frac{1-k}{2}=1
$$

which is impossible. Consequently, $\mu_{A}(x y) \geq \min \left\{\mu_{A}(x), \mu_{A}(y), \frac{1-k}{2}\right\}$ and $\gamma_{A}(x y) \leq$ $\max \left\{\gamma_{A}(x), \gamma_{A}(y), \frac{1-k}{2}\right\}$ for all $x, y \in S$. Let $a, b, c \in S$ be such that $\min \left\{\mu_{A}(a), \mu_{A}(c)\right\}<\frac{1-k}{2}$ and $\max \left\{\gamma_{A}(a), \gamma_{A}(c)\right\}>\frac{1-k}{2}$. We claim that

$$
\mu_{A}(a b c) \geq \min \left\{\mu_{A}(a), \mu_{A}(c)\right\} \quad \text { and } \quad \gamma_{A}(a b c) \leq \max \left\{\gamma_{A}(a), \gamma_{A}(c)\right\}
$$

If not, then $\mu_{A}(a b c)<t_{0} \leq \min \left\{\mu_{A}(a), \mu_{A}(c)\right\}$ and $\gamma_{A}(a b c)>s_{0} \geq \max \left\{\gamma_{A}(a), \gamma_{A}(c)\right\}$ for some $t_{0} \in\left(0, \frac{1-k}{2}\right)$ and $s_{0} \in\left(\frac{1-k}{2}, 1\right)$. It follows that $\left\langle a ;\left(t_{0}, s_{0}\right)\right\rangle \in A$ and $\left\langle c ;\left(t_{0}, s_{0}\right)\right\rangle \in$ $A$, but $\left\langle a b c ;\left(t_{0}, s_{0}\right)\right\rangle \bar{\epsilon} A$ and $\mu_{A}(a b c)+t_{0}<2 t_{0}<1-k$ and $\gamma_{A}(a b c)+s_{0}>2 s_{0}>1-$ $k$, i.e., $\left\langle a b c ;\left(t_{0}, s_{0}\right)\right\rangle \overline{\mathrm{q}_{k}} A$. This is a contradiction. Thus, $\mu_{A}(a b c) \geq \min \left\{\mu_{A}(a), \mu_{A}(c)\right\}$ and $\gamma_{A}(a b c) \leq \max \left\{\gamma_{A}(a), \gamma_{A}(c)\right\}$ for all $a, b, c \in S$ with $\min \left\{\mu_{A}(a), \mu_{A}(c)\right\}<\frac{1-k}{2}$ and 
$\max \left\{\gamma_{A}(a), \gamma_{A}(c)\right\}>\frac{1-k}{2}$. If $\min \left\{\mu_{A}(a), \mu_{A}(c)\right\} \geq \frac{1-k}{2}$ and $\max \left\{\gamma_{A}(a), \gamma_{A}(c)\right\} \leq \frac{1-k}{2}$, then $\left\langle a ;\left(\frac{1-k}{2}, \frac{1-k}{2}\right)\right\rangle \in A$ and $\left\langle c ;\left(\frac{1-k}{2}, \frac{1-k}{2}\right)\right\rangle \in A$. Using (q3), we have

$$
\begin{aligned}
& \left\langle a b c ;\left(\frac{1-k}{2}, \frac{1-k}{2}\right)\right\rangle \\
& \quad=\left\langle a b c ; \min \left\{\frac{1-k}{2}, \frac{1-k}{2}\right\}, \max \left\{\frac{1-k}{2}, \frac{1-k}{2}\right\}\right\} \in \vee \mathrm{q} A,
\end{aligned}
$$

and so $\mu_{A}(a b c) \geq \frac{1-k}{2}$ and $\gamma_{A}(a b c) \leq \frac{1-k}{2}$ or $\mu_{A}(a b c)+\frac{1-k}{2}>1$ and $\gamma_{A}(a b c)+\frac{1-k}{2}<1$. If $\mu_{A}(a b c)<\frac{1-k}{2}$ and $\gamma_{A}(a b c)>\frac{1-k}{2}$, then

$$
\mu_{A}(a b c)+\frac{1-k}{2}<\frac{1-k}{2}+\frac{1-k}{2}=1
$$

and

$$
\gamma_{A}(a b c)+\frac{1-k}{2}>\frac{1-k}{2}+\frac{1-k}{2}=1
$$

which is impossible. Therefore, $\mu_{A}(x y z) \geq \min \left\{\mu_{A}(x), \mu_{A}(z), \frac{1-k}{2}\right\}$ and $\gamma_{A}(x y z) \leq$ $\max \left\{\gamma_{A}(x), \gamma_{A}(z), \frac{1-k}{2}\right\}$ for all $x, y, z \in S$.

Conversely, let $A=\left\langle x, \mu_{A}, \gamma_{A}\right\rangle$ be an IFS of $S$ that satisfies the three conditions (1), (2) and (3). Let $x, y \in S, t \in(0,1]$ and $s \in[0,1)$ be such that $x \leq y$ and $[y ;(t, s)] \in A$. Then $\mu_{A}(y) \geq t$ and $\gamma_{A}(y) \leq s$, and so,

$$
\begin{aligned}
\mu_{A}(x) & \geq \min \left\{\mu_{A}(y), \frac{1-k}{2}\right\} \geq \min \left\{t, \frac{1-k}{2}\right\} \\
& = \begin{cases}t & \text { if } t \leq \frac{1-k}{2}, \\
\frac{1-k}{2} & \text { if } t>\frac{1-k}{2}\end{cases}
\end{aligned}
$$

and

$$
\begin{aligned}
\gamma_{A}(x) & \leq \max \left\{\gamma_{A}(y), \frac{1-k}{2}\right\} \leq \max \left\{s, \frac{1-k}{2}\right\} \\
& = \begin{cases}s & \text { if } s \geq \frac{1-k}{2}, \\
\frac{1-k}{2} & \text { if } s<\frac{1-k}{2} .\end{cases}
\end{aligned}
$$

It follows that $\mu_{A}(x) \geq t$ and $\gamma_{A}(x) \leq s$ or $\mu_{A}(x)+t \geq \frac{1-k}{2}+t>1-k$ and $\gamma_{A}(x)+s \leq \frac{1-k}{2}+s<$ $1-k$, i.e., $\langle x ;(t, s)\rangle \in A$ or $\langle x ;(t, s)\rangle \mathrm{q}_{k} A$. Hence, $\langle x ;(t, s)\rangle \in \vee \mathrm{q}_{k} A$. Let $x, y \in S, t_{1}, t_{2} \in(0,1]$ and $s_{1}, s_{2} \in[0,1)$ be such that $\left\langle x ;\left(t_{1}, s_{1}\right)\right\rangle \in A$ and $\left\langle y ;\left(t_{2}, s_{2}\right)\right\rangle \in A$. Then $\mu_{A}(x) \geq t_{1}, \gamma_{A}(x) \leq s_{1}$ and $\mu_{A}(y) \geq t_{2}, \gamma_{A}(y) \leq s_{2}$. It follows from (2) that

$$
\begin{aligned}
\mu_{A}(x y) & \geq \min \left\{\mu_{A}(x), \mu_{A}(y), \frac{1-k}{2}\right\} \geq \min \left\{t_{1}, t_{2}, \frac{1-k}{2}\right\} \\
& = \begin{cases}\min \left\{t_{1}, t_{2}\right\} & \text { if } \min \left\{t_{1}, t_{2}\right\} \leq \frac{1-k}{2}, \\
\frac{1-k}{2} & \text { if } \min \left\{t_{1}, t_{2}\right\} \leq \frac{1-k}{2}\end{cases}
\end{aligned}
$$


and

$$
\begin{aligned}
\gamma_{A}(x y) & \leq \max \left\{\gamma_{A}(x), \gamma_{A}(y), \frac{1-k}{2}\right\} \leq \max \left\{s_{1}, s_{2}, \frac{1-k}{2}\right\} \\
& = \begin{cases}\max \left\{s_{1}, s_{2}\right\} & \text { if } \max \left\{s_{1}, s_{2}\right\} \geq \frac{1-k}{2} \\
\frac{1-k}{2} & \text { if } \max \left\{s_{1}, s_{2}\right\}<\frac{1-k}{2} .\end{cases}
\end{aligned}
$$

It follows that $\left\langle x y ; \min \left\{t_{1}, t_{2}\right\}, \max \left\{s_{1}, s_{2}\right\}\right\rangle \in A$ or $\mu_{A}(x y)+\min \left\{t_{1}, t_{2}\right\} \geq \frac{1-k}{2}+\min \left\{t_{1}, t_{2}\right\}>$ $\frac{1-k}{2}+\frac{1-k}{2}=1-k$ and $\gamma_{A}(x y)+\max \left\{s_{1}, s_{2}\right\} \leq \frac{1-k}{2}+\max \left\{s_{1}, s_{2}\right\}<\frac{1-k}{2}+\frac{1-k}{2}=1-k$, i.e., $\left\langle x y ; \min \left\{t_{1}, t_{2}\right\}, \max \left\{s_{1}, s_{2}\right\}\right\rangle \mathrm{q}_{k} A$. Therefore, $\left\langle x y ; \min \left\{t_{1}, t_{2}\right\}, \max \left\{s_{1}, s_{2}\right\}\right\rangle \in \vee \mathrm{q}_{k} A$. Let $x, y, z \in$ $S, t_{1}, t_{2} \in(0,1]$ and $s_{1}, s_{2} \in[0,1)$ be such that $\left\langle x ;\left(t_{1}, s_{1}\right)\right\rangle \in A$ and $\left\langle z ;\left(t_{2}, s_{2}\right)\right\rangle \in A$. Then $\mu_{A}(x) \geq t_{1}, \gamma_{A}(x) \leq s_{1}$ and $\mu_{A}(z) \geq t_{2}, \gamma_{A}(z) \leq s_{2}$. It follows from (3) that

$$
\begin{aligned}
\mu_{A}(x y z) & \geq \min \left\{\mu_{A}(x), \mu_{A}(z), \frac{1-k}{2}\right\} \geq \min \left\{t_{1}, t_{2}, \frac{1-k}{2}\right\} \\
& = \begin{cases}\min \left\{t_{1}, t_{2}\right\} & \text { if } \min \left\{t_{1}, t_{2}\right\} \leq \frac{1-k}{2} \\
\frac{1-k}{2} & \text { if } \min \left\{t_{1}, t_{2}\right\} \leq \frac{1-k}{2}\end{cases}
\end{aligned}
$$

and

$$
\begin{aligned}
\gamma_{A}(x y z) & \leq \max \left\{\gamma_{A}(x), \gamma_{A}(z), \frac{1-k}{2}\right\} \leq \max \left\{s_{1}, s_{2}, \frac{1-k}{2}\right\} \\
& = \begin{cases}\max \left\{s_{1}, s_{2}\right\} & \text { if } \max \left\{s_{1}, s_{2}\right\} \geq \frac{1-k}{2}, \\
\frac{1-k}{2} & \text { if } \max \left\{s_{1}, s_{2}\right\}<\frac{1-k}{2} .\end{cases}
\end{aligned}
$$

Thus, we have $\left\langle x y z ; \min \left\{t_{1}, t_{2}\right\}, \max \left\{s_{1}, s_{2}\right\}\right\rangle \in A$ or $\mu_{A}(x y z)+\min \left\{t_{1}, t_{2}\right\} \geq \frac{1-k}{2}+$ $\min \left\{t_{1}, t_{2}\right\}>\frac{1-k}{2}+\frac{1-k}{2}=1-k$ and $\gamma_{A}(x y z)+\max \left\{s_{1}, s_{2}\right\} \leq \frac{1-k}{2}+\max \left\{s_{1}, s_{2}\right\}<\frac{1-k}{2}+\frac{1-k}{2}=1-k$, i.e., $\left\langle x y z ; \min \left\{t_{1}, t_{2}\right\}, \max \left\{s_{1}, s_{2}\right\}\right\rangle \mathrm{q}_{k} A$. Therefore, $\left\langle x y z ; \min \left\{t_{1}, t_{2}\right\}, \max \left\{s_{1}, s_{2}\right\}\right\rangle \in \vee \mathrm{q}_{k} A$. Thus, $A=\left\langle x, \mu_{A}, \gamma_{A}\right\rangle$ is an $\left(\in, \in \vee \mathrm{q}_{k}\right)$-intuitionistic fuzzy bi-ideal of $S$.

If we take $k=0$ in Theorem 3.5, then we have the following corollary.

Corollary 3.6 [11, Theorem 3.5] An IFS $A=\left\langle x, \mu_{A}, \gamma_{A}\right\rangle$ of $S$ is an $\left(\in, \in \vee \mathrm{q}_{k}\right)$-intuitionistic fuzzy bi-ideal of $S$ if and only if it satisfies the conditions

$$
\begin{array}{ll}
\text { (1) } & \left(\begin{array}{l}
x \leq y \Longrightarrow \mu_{A}(x) \geq \min \left\{\mu_{A}(y), 0.5\right\} \\
\text { and } \gamma_{A}(x) \leq \max \left\{\gamma_{A}(y), 0.5\right\}
\end{array}\right), \\
\text { (2) }\left(\begin{array}{l}
\mu_{A}(x y) \geq \min \left\{\mu_{A}(x), \mu_{A}(y), 0.5\right\} \\
\text { and } \gamma_{A}(x y) \leq \max \left\{\gamma_{A}(x), \gamma_{A}(y), 0.5\right\}
\end{array}\right), \\
\text { (3) }\left(\begin{array}{l}
\mu_{A}(x y z) \geq \min \left\{\mu_{A}(x), \mu_{A}(z), 0.5\right\} \\
\text { and } \gamma_{A}(x y z) \leq \max \left\{\gamma_{A}(x), \gamma_{A}(z), 0.5\right\}
\end{array}\right) .
\end{array}
$$

Obviously, every intuitionistic fuzzy bi-ideal is an $(\epsilon, \epsilon)$-intuitionistic fuzzy bi-ideal, and we know that every $(\epsilon, \epsilon)$-intuitionistic fuzzy bi-ideal of $S$ is an $(\epsilon, \in \vee$ q)-intuitionistic 
fuzzy bi-ideal of $S$, and every $(\in, \in \vee \mathrm{q})$-intuitionistic fuzzy bi-ideal is an $\left(\in, \in \vee \mathrm{q}_{k}\right)$ intuitionistic fuzzy bi-ideal of $S$. But the converse may not be true. The following example shows that every $\left(\in, \in \vee \mathrm{q}_{k}\right)$-intuitionistic fuzzy bi-ideal of $S$ may not be an $(\in, \in \vee \mathrm{q})$ intuitionistic fuzzy bi-ideal nor an intuitionistic fuzzy bi-ideal of $S$.

Example 3.7 Consider the ordered semigroup of Example 3.4 and define an IFS $A=$ $\left\langle x, \mu_{A}, \gamma_{A}\right\rangle$ by

$$
\mu_{A}: S \rightarrow[0,1] \mid \mu_{A}(x)= \begin{cases}0.80 & \text { if } x=a, \\ 0.60 & \text { if } x=b, \\ 0.40 & \text { if } x=c \\ 0.30 & \text { if } x=d, e\end{cases}
$$

and

$$
\gamma_{A}: S \rightarrow[0,1] \mid \gamma_{A}(x)= \begin{cases}0.20 & \text { if } x=a, \\ 0.30 & \text { if } x=b, \\ 0.40 & \text { if } x=c, \\ 0.70 & \text { if } x=d, e .\end{cases}
$$

Then $A=\left\langle x, \mu_{A}, \gamma_{A}\right\rangle$ is an $\left(\in, \in \vee \mathrm{q}_{0.4}\right)$-intuitionistic fuzzy bi-ideal of $S$. But

(1) $A=\left\langle x, \mu_{A}, \gamma_{A}\right\rangle$ is not an $(\in, \in \vee \mathrm{q})$-intuitionistic fuzzy bi-ideal of $S$. Since $\langle a ;(0.8,0.2)\rangle \in A$ and $\langle b ;(0.6,0.3)\rangle \in A$ but $\langle a b ;(0.6,0.3)\rangle \in \overline{\epsilon \vee} A$.

(2) $A=\left\langle x, \mu_{A}, \gamma_{A}\right\rangle$ is not an intuitionistic fuzzy bi-ideal of $S$. Since

$$
\mu_{A}(a b)=\mu_{A}(d)=0.30<m\left\{\mu_{A}(a)=0.80, \mu_{A}(b)=0.60\right\}
$$

and

$$
\gamma_{A}(a b)=\gamma_{A}(d)=0.70>M\left\{\gamma_{A}(a)=0.20, \mu_{A}(b)=0.30\right\}
$$

In the following, we give a condition for an $\left(\in, \in \vee \mathrm{q}_{k}\right)$-intuitionistic fuzzy bi-ideal of $S$ to be an ordinary intuitionistic fuzzy bi-ideal of $S$.

Theorem 3.8 Let $A=\left\langle x, \mu_{A}, \gamma_{A}\right\rangle$ be an $\left(\in, \in \vee \mathrm{q}_{k}\right)$-intuitionistic fuzzy bi-ideal of $S$. If $\mu_{A}(x) \geq \frac{1-k}{2}$ and $\gamma_{A}(x) \leq \frac{1-k}{2}$ for all $x \in S$, then $A=\left\langle x, \mu_{A}, \gamma_{A}\right\rangle$ is an $(\in, \in)$-intuitionistic fuzzy bi-ideal of $S$.

Proof The proof is straightforward by Theorem 3.5 .

Corollary 3.9 [11, Theorem 3.8] Let $A=\left\langle x, \mu_{A}, \gamma_{A}\right\rangle$ be an $(\in, \in \vee \mathrm{q})$-intuitionistic fuzzy bi-ideal of $S$. If $\mu_{A}(x) \geq 0.5$ and $\gamma_{A}(x) \leq 0.5$ for all $x \in S$, then $A=\left\langle x, \mu_{A}, \gamma_{A}\right\rangle$ is an $(\in, \in)$ intuitionistic fuzzy bi-ideal of $S$.

Proof The proof follows from Theorem 3.8, by taking $k=0$. 
Theorem 3.10 For an IFS $A=\left\langle x, \mu_{A}, \gamma_{A}\right\rangle$ of $S$, the following are equivalent:

(1) $A=\left\langle x, \mu_{A}, \gamma_{A}\right\rangle$ is an $\left(\in, \in \vee \mathrm{q}_{k}\right)$-intuitionistic fuzzy bi-ideal of $S$.

(2) $\left(\forall t \in\left(0, \frac{1-k}{2}\right]\right)\left(\forall s \in\left[\frac{1-k}{2}, 1\right)\right)\left(C_{(\alpha, \beta)}(A) \neq \emptyset \Longrightarrow C_{(\alpha, \beta)}(A)\right.$ is a bi-ideal of $\left.S\right)$.

Proof Assume that $A=\left\langle x, \mu_{A}, \gamma_{A}\right\rangle$ is an $\left(\in, \in \vee \mathrm{q}_{k}\right)$-intuitionistic fuzzy bi-ideal of $S$, let $t \in\left(0, \frac{1-k}{2}\right]$ and $s \in\left[\frac{1-k}{2}, 1\right)$ be such that $C_{(\alpha, \beta)}(A) \neq \emptyset$. Using Theorem 3.5(1), we have

$$
\mu_{A}(x) \geq \min \left\{\mu_{A}(y), \frac{1-k}{2}\right\} \quad \text { and } \quad \gamma_{A}(x) \leq \max \left\{\gamma_{A}(y), \frac{1-k}{2}\right\}
$$

for any $x, y \in S$ with $x \leq y$ and $x \in C_{(t, s)}(A)$. It follows that $\mu_{A}(x) \geq \min \{t, 0.5\}=t$ and $\gamma_{A}(x) \leq \max \{s, 0.5\}=s$, so that $y \in C_{(t, s)}(A)$. Let $x, y \in C_{(\alpha, \beta)}(A)$. Then $\mu_{A}(x) \geq t, \gamma_{A}(x) \leq s$ and $\mu_{A}(y) \geq t, \gamma_{A}(y) \leq s$. Theorem 3.5(2) implies that

$$
\mu_{A}(x y) \geq \min \left\{\mu_{A}(x), \mu_{A}(y), \frac{1-k}{2}\right\} \geq \min \left\{t, \frac{1-k}{2}\right\}=t
$$

and

$$
\gamma_{A}(x y) \leq \max \left\{\gamma_{A}(x), \gamma_{A}(y), \frac{1-k}{2}\right\} \leq \max \left\{s, \frac{1-k}{2}\right\}=s .
$$

Thus, $x y \in C_{(\alpha, \beta)}(A)$. Now let $x, z \in C_{(\alpha, \beta)}(A)$. Then $\mu_{A}(x) \geq t, \gamma_{A}(x) \leq s$ and $\mu_{A}(z) \geq t$, $\gamma_{A}(z) \leq s$. Theorem 3.5(3) induces that

$$
\mu_{A}(x y z) \geq \min \left\{\mu_{A}(x), \mu_{A}(z), \frac{1-k}{2}\right\} \geq \min \left\{t, \frac{1-k}{2}\right\}=t
$$

and

$$
\gamma_{A}(x y z) \leq \max \left\{\gamma_{A}(x), \gamma_{A}(z), \frac{1-k}{2}\right\} \leq \max \left\{s, \frac{1-k}{2}\right\}=s .
$$

Thus, $x y z \in C_{(\alpha, \beta)}(A)$, therefore, $C_{(\alpha, \beta)}(A)$ is a bi-ideal of $S$.

Conversely, let $A=\left\langle x, \mu_{A}, \gamma_{A}\right\rangle$ be an IFS of $S$ such that $C_{(\alpha, \beta)}(A)$ is non-empty and is a bi-ideal of $S$ for all $t \in\left(0, \frac{1-k}{2}\right]$ and $s \in\left[\frac{1-k}{2}, 1\right)$. If there exist $a, b \in S$ with $a \leq b$ and $b \in C_{(\alpha, \beta)}(A)$ such that $\mu_{A}(a)<\min \left\{\mu_{A}(b), \frac{1-k}{2}\right\}$ and $\gamma_{A}(a)>\max \left\{\gamma_{A}(b), \frac{1-k}{2}\right\}$, then $\mu_{A}(a)<t_{a} \leq \min \left\{\mu_{A}(b), \frac{1-k}{2}\right\}$ and $\gamma_{A}(a)>s_{a} \geq \max \left\{\gamma_{A}(b), \frac{1-k}{2}\right\}$ for some $t_{a} \in\left(0, \frac{1-k}{2}\right]$ and $s_{a} \in\left[\frac{1-k}{2}, 1\right)$. Then $a \notin C_{\left(t_{a}, s_{a}\right)}(A)$, a contradiction. Therefore, $\mu_{A}(x) \geq \min \left\{\mu_{A}(y), \frac{1-k}{2}\right\}$ and $\gamma_{A}(x)>\max \left\{\gamma_{A}(y), \frac{1-k}{2}\right\}$ for all $x, y \in S$ with $x \leq y$. Assume that there exist $a, b \in S$ such that

$$
\mu_{A}(a b)<\min \left\{\mu_{A}(a), \mu_{A}(b), \frac{1-k}{2}\right\}
$$

and $\gamma_{A}(a b)>\max \left\{\gamma_{A}(a), \gamma_{A}(b), \frac{1-k}{2}\right\}$. Then

$$
\mu_{A}(a b)<t_{0} \leq \min \left\{\mu_{A}(a), \mu_{A}(b), \frac{1-k}{2}\right\}
$$

and

$$
\gamma_{A}(a b)>s_{0} \geq \max \left\{\gamma_{A}(a), \gamma_{A}(b), \frac{1-k}{2}\right\}
$$


for some $t_{0} \in\left(0, \frac{1-k}{2}\right]$ and $s_{0} \in\left[\frac{1-k}{2}, 1\right)$. It follows that $a \in C_{\left(t_{0}, s_{0}\right)}(A)$ and $b \in C_{\left(t_{0}, s_{0}\right)}(A)$, but $a b \notin C_{\left(t_{0}, s_{0}\right)}(A)$. This is a contradiction. Hence

$$
\mu_{A}(x y) \geq \min \left\{\mu_{A}(x), \mu_{A}(y), \frac{1-k}{2}\right\}
$$

and

$$
\gamma_{A}(x y) \leq \max \left\{\gamma_{A}(x), \gamma_{A}(y), \frac{1-k}{2}\right\}
$$

for all $x, y \in S$. Suppose that

$$
\mu_{A}(a b c)<\min \left\{\mu_{A}(a), \mu_{A}(c), \frac{1-k}{2}\right\}
$$

and

$$
\gamma_{A}(a b c)>\max \left\{\gamma_{A}(a), \gamma_{A}(c), \frac{1-k}{2}\right\}
$$

for some $a, b, c \in S$. Then there exist $t_{1} \in\left(0, \frac{1-k}{2}\right]$ and $s_{1} \in\left[\frac{1-k}{2}, 1\right)$ such that

$$
\mu_{A}(a b c)<t_{1} \leq \min \left\{\mu_{A}(a), \mu_{A}(c), \frac{1-k}{2}\right\}
$$

and

$$
\gamma_{A}(a b c)>s_{1} \geq \max \left\{\gamma_{A}(a), \gamma_{A}(c), \frac{1-k}{2}\right\}
$$

Then $a \in C_{\left(t_{1}, s_{1}\right)}(A)$ and $c \in C_{\left(t_{1}, s_{1}\right)}(A)$, but $a b c \notin C_{\left(t_{1}, s_{1}\right)}(A)$. This is impossible, and hence $\mu_{A}(x y z)<\min \left\{\mu_{A}(x), \mu_{A}(z), \frac{1-k}{2}\right\}$ and

$$
\gamma_{A}(x y z)>\max \left\{\gamma_{A}(x), \gamma_{A}(z), \frac{1-k}{2}\right\}
$$

for all $x, y, z \in S$. Therefore, $A=\left\langle x, \mu_{A}, \gamma_{A}\right\rangle$ is an $\left(\in, \in \vee \mathrm{q}_{k}\right)$-intuitionistic fuzzy bi-ideal of $S$.

By taking $k=0$ in Theorem 3.10, we get the following corollary.

Corollary 3.11 [11, Theorem 3.10] For an IFS $A=\left\langle x, \mu_{A}, \gamma_{A}\right\rangle$ of an ordered semigroup $(S, \cdot, \leq)$, the following are equivalent

(1) $A=\left\langle x, \mu_{A}, \gamma_{A}\right\rangle$ is an $(\in, \in \vee \mathrm{q})$-intuitionistic fuzzy bi-ideal of $S$.

(2) $(\forall t \in(0,0.5])(\forall s \in[0.5,1))\left(C_{(\alpha, \beta)}(A) \neq \emptyset \Longrightarrow C_{(\alpha, \beta)}(A)\right.$ is a bi-ideal of $\left.S\right)$.

For an IFP $\langle x ;(\alpha, \beta)\rangle$ of $S$ and an IFS $A=\left\langle x, \mu_{A}, \gamma_{A}\right\rangle$ of $S$, we say that

(c4) $\langle x ;(\alpha, \beta)\rangle \mathrm{q} A$ if $\mu_{A}(x)+\alpha \geq 1$ and $\gamma_{A}(x)+\beta \leq 1$,

(c5) $\langle x ;(\alpha, \beta)\rangle \underline{\mathrm{q}_{k}} A$ if $\mu_{A}(x)+\alpha+k \geq 1$ and $\gamma_{A}(x)+\beta+k \leq 1$. 
We denote by $Q_{(\alpha, \beta)}^{k}(A)$ (resp. $\left.\underline{Q}_{(\alpha, \beta)}^{k}(A)\right)$ the set $\left\{x \in S \mid\langle x ;(\alpha, \beta)\rangle \mathrm{q}_{k} A\right\}$ (resp. $\{x \in$

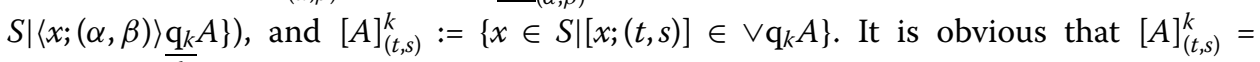
$C_{(t, s)}(A) \cup \overline{Q_{(t, s)}^{k}}(A)$.

Proposition 3.12 If $A=\left\langle x, \mu_{A}, \gamma_{A}\right\rangle$ is an $\left(\in, \in \vee \mathrm{q}_{k}\right)$-intuitionistic fuzzy bi-ideal of $S$, then

$$
\left(\forall t \in\left(\frac{1-k}{2}, 1\right]\right)\left(\forall s \in\left[0, \frac{1-k}{2}\right)\right) \quad\left(Q_{(t, s)}^{k}(A) \neq \emptyset \Longrightarrow Q_{(t, s)}^{k}(A) \text { is a bi-ideal of } S\right) .
$$

Proof Assume that $A=\left\langle x, \mu_{A}, \gamma_{A}\right\rangle$ is an $\left(\in, \in \vee \mathrm{q}_{k}\right)$-intuitionistic fuzzy bi-ideal of $S$. Let $t \in\left[\frac{1-k}{2}, 1\right]$ and $s \in\left[0, \frac{1-k}{2}\right]$ be such that $Q_{(t, s)}^{k}(A) \neq \emptyset$. Let $y \in Q_{(t, s)}^{k}(A)$ and $x \in S$ be such that $x \leq y$. Then $\mu_{A}(y)+t+k>1$ and $\gamma_{A}(y)+s+k<1$. By means of Theorem 3.5(1), we have

$$
\begin{aligned}
\mu_{A}(x) & \geq \min \left\{\mu_{A}(y), \frac{1-k}{2}\right\} \\
& = \begin{cases}\frac{1-k}{2} & \text { if } \mu_{A}(y) \geq \frac{1-k}{2}, \\
\mu_{A}(y) & \text { if } \mu_{A}(y)<\frac{1-k}{2}\end{cases} \\
& >1-t-k
\end{aligned}
$$

and

$$
\begin{aligned}
\gamma_{A}(x) & \leq \max \left\{\gamma_{A}(y), \frac{1-k}{2}\right\} \\
& = \begin{cases}\frac{1-k}{2} & \text { if } \gamma_{A}(y)<\frac{1-k}{2}, \\
\gamma_{A}(y) & \text { if } \gamma_{A}(y) \geq \frac{1-k}{2}\end{cases} \\
& <1-s-k .
\end{aligned}
$$

It follows that $x \in Q_{(t, s)}(A)$. Let $x, y \in Q_{(t, s)}(A)$. Then $\mu_{A}(x)+t>1-k$ and $\gamma_{A}(x)+s<1-k$, $\mu_{A}(y)+t>1-k$ and $\gamma_{A}(y)+s<1-k$. Using (2) of Theorem 3.5, we have that

$$
\begin{aligned}
\mu_{A}(x y) & \geq \min \left\{\mu_{A}(x), \mu_{A}(y), \frac{1-k}{2}\right\} \\
& = \begin{cases}\frac{1-k}{2} & \text { if } \min \left\{\mu_{A}(x), \mu_{A}(y)\right\} \geq \frac{1-k}{2}, \\
\min \left\{\mu_{A}(x), \mu_{A}(y)\right\} & \text { if } \min \left\{\mu_{A}(x), \mu_{A}(y)\right\}<\frac{1-k}{2}\end{cases} \\
& >1-t-k,
\end{aligned}
$$

and

$$
\begin{aligned}
& \gamma_{A}(x y) \leq \max \left\{\gamma_{A}(x), \gamma_{A}(y), \frac{1-k}{2}\right\} \\
& = \begin{cases}\frac{1-k}{2} & \text { if } \max \left\{\gamma_{A}(x), \gamma_{A}(y)\right\}<\frac{1-k}{2}, \\
\max \left\{\gamma_{A}(x), \gamma_{A}(y)\right\} & \text { if } \max \left\{\gamma_{A}(x), \gamma_{A}(y)\right\} \geq \frac{1-k}{2}\end{cases} \\
& <1-s-k \text {. }
\end{aligned}
$$


Thus, $x y \in Q_{(t, s)}(A)$. Let $x, z \in Q_{(t, s)}(A)$ and $y \in S$. Then $\mu_{A}(x)+t>1-k$ and $\gamma_{A}(x)+s<$ $1-k, \mu_{A}(z)+t>1-k$ and $\gamma_{A}(z)+s<1-k$. Using (3) of Theorem 3.5, we have that

$$
\begin{aligned}
\mu_{A}(x y z) & \geq \min \left\{\mu_{A}(x), \mu_{A}(z), \frac{1-k}{2}\right\} \\
& = \begin{cases}\frac{1-k}{2} & \text { if } \min \left\{\mu_{A}(x), \mu_{A}(z)\right\} \geq \frac{1-k}{2}, \\
\min \left\{\mu_{A}(x), \mu_{A}(z)\right\} & \text { if } \min \left\{\mu_{A}(x), \mu_{A}(z)\right\}<\frac{1-k}{2}\end{cases} \\
& >1-t-k
\end{aligned}
$$

and

$$
\begin{aligned}
\gamma_{A}(x y z) & \leq \max \left\{\gamma_{A}(x), \gamma_{A}(z), \frac{1-k}{2}\right\} \\
& = \begin{cases}\frac{1-k}{2} & \text { if } \max \left\{\gamma_{A}(x), \gamma_{A}(z)\right\}<\frac{1-k}{2}, \\
\max \left\{\gamma_{A}(x), \gamma_{A}(z)\right\} & \text { if } \max \left\{\gamma_{A}(x), \gamma_{A}(z)\right\} \geq \frac{1-k}{2}\end{cases} \\
& <1-s .
\end{aligned}
$$

Hence $x y z \in Q_{(t, s)}^{k}(A)$. Therefore, $Q_{(t, s)}^{k}(A)$ is a bi-ideal of $S$.

Theorem 3.13 For any IFS $A=\left\langle x, \mu_{A}, \gamma_{A}\right\rangle$ of $S$, the following are equivalent

(1) $A=\left\langle x, \mu_{A}, \gamma_{A}\right\rangle$ is an $\left(\in, \in \vee \mathrm{q}_{k}\right)$-intuitionistic fuzzy bi-ideal of $S$.

(2) $(\forall t \in(0,1])(\forall s \in[0,1))\left([A]_{(t, s)}^{k} \neq \emptyset \Longrightarrow[A]_{(t, s)}^{k}\right.$ is a bi-ideal of $\left.S\right)$.

We call $[A]_{(t, s)}^{k}$ an $(\in \vee \mathrm{q})$-level bi-ideal of $A=\left\langle x, \mu_{A}, \gamma_{A}\right\rangle$.

Proof Assume that $A=\left\langle x, \mu_{A}, \gamma_{A}\right\rangle$ is an $\left(\in, \in \vee \mathrm{q}_{k}\right)$-intuitionistic fuzzy bi-ideal of $S$, and let $t \in(0,1]$ and $s \in[0,1)$ be such that $[A]_{(t, s)}^{k} \neq \emptyset$. Let $y \in[A]_{(t, s)}^{k}$ and $x \in S$ be such that $x \leq y$. Then $y \in C_{(t, s)}(A)$ or $y \in Q_{(t, s)}^{k}(A)$, i.e., $\mu_{A}(y) \geq t$ and $\gamma_{A}(y) \leq s$ or $\mu_{A}(y)+t>1-k$ and $\gamma_{A}(y)+s<1-k$. Using Theorem 3.5(1), we get

$$
\mu_{A}(x) \geq \min \left\{\mu_{A}(y), \frac{1-k}{2}\right\} \quad \text { and } \quad \gamma_{A}(x) \leq \max \left\{\gamma_{A}(y), \frac{1-k}{2}\right\}
$$

We consider two cases $\mu_{A}(y) \leq \frac{1-k}{2}, \gamma_{A}(y) \geq \frac{1-k}{2}$ and $\mu_{A}(y)>\frac{1-k}{2}, \gamma_{A}(y)<\frac{1-k}{2}$. The first case implies from (3.1) that $\mu_{A}(x) \geq \mu_{A}(y)$ and $\gamma_{A}(x) \leq \gamma_{A}(y)$. Thus, if $\mu_{A}(y) \geq t$ and $\gamma_{A}(y) \leq$ $s$, then $\mu_{A}(x) \geq t$ and $\gamma_{A}(x) \leq s$, and so, $x \in C_{(t, s)}(A) \subseteq[A]_{(t, s)}^{k}$. If $\mu_{A}(y)+t>1-k$ and $\gamma_{A}(y)+$ $s<1-k$, then $\mu_{A}(x)+t \geq \mu_{A}(y)+t>1-k$ and $\gamma_{A}(x)+s \leq \gamma_{A}(y)+s<1-k$, which implies that $[x ;(t, s)] \mathrm{q}_{k} A$, i.e., $x \in Q_{(t, s)}^{k}(A) \subseteq[A]_{(t, s)}^{k}$. Combining the second case and (3.1), we have $\mu_{A}(x) \geq \frac{1-k}{2}$ and $\gamma_{A}(x) \leq \frac{1-k}{2}$. If $t \leq \frac{1-k}{2}$ and $s \geq \frac{1-k}{2}$, then $\mu_{A}(x) \geq t$ and $\gamma_{A}(x) \leq s$, and hence $x \in C_{(t, s)}(A) \subseteq[A]_{(t, s)}^{k}$. If $t>\frac{1-k}{2}$ and $s<\frac{1-k}{2}$, then $\mu_{A}(x)+t>\frac{1-k}{2}+\frac{1-k}{2}=1-k$ and $\gamma_{A}(x)+$ $s<\frac{1-k}{2}+\frac{1-k}{2}=1-k$, which implies that $x \in Q_{(t, s)}^{k}(A) \subseteq[A]_{(t, s)}^{k}$. Therefore, $[A]_{(t, s)}^{k}$ satisfies the condition (b1). Let $x, y \in[A]_{(t, s)}^{k}$. Then $x \in C_{(t, s)}(A)$ or $\langle x ;(t, s)\rangle \mathrm{q}_{k} A$ and $y \in C_{(t, s)}(A)$ or $\langle y ;(t, s)\rangle \mathrm{q}_{k} A$, that is, $\mu_{A}(x) \geq t, \gamma_{A}(x) \leq s$ or $\mu_{A}(x)+t+k>1, \gamma_{A}(x)+s+k<1$ and $\mu_{A}(y) \geq t$, $\gamma_{A}(y) \leq s$ or $\mu_{A}(y)+t+k>1, \gamma_{A}(y)+s+k<1$. We consider the following four cases

(i) $\mu_{A}(x) \geq t, \gamma_{A}(x) \leq s$ and $\mu_{A}(y) \geq t, \gamma_{A}(y) \leq s$,

(ii) $\mu_{A}(x) \geq t, \gamma_{A}(x) \leq s$ and $\mu_{A}(y)+t+k>1, \gamma_{A}(y)+s+k<1$, 
(iii) $\mu_{A}(x)+t+k>1, \gamma_{A}(x)+s+k<1$ and $\mu_{A}(y) \geq t, \gamma_{A}(y) \leq s$,

(iv) $\mu_{A}(x)+t+k>1, \gamma_{A}(x)+s+k<1$ and $\mu_{A}(y)+t+k>1, \gamma_{A}(y)+s+k<1$.

For the case (i), Theorem 3.5(2) implies that

$$
\begin{aligned}
\mu_{A}(x y) & \geq \min \left\{\mu_{A}(x), \mu_{A}(y), \frac{1-k}{2}\right\} \geq \min \left\{t, \frac{1-k}{2}\right\} \\
& = \begin{cases}\frac{1-k}{2} & \text { if } t>\frac{1-k}{2}, \\
t & \text { if } t \leq \frac{1-k}{2}\end{cases}
\end{aligned}
$$

and

$$
\begin{aligned}
\gamma_{A}(x y) & \leq \max \left\{\gamma_{A}(x), \gamma_{A}(y), \frac{1-k}{2}\right\} \leq \max \left\{s, \frac{1-k}{2}\right\} \\
& = \begin{cases}\frac{1-k}{2} & \text { if } s<\frac{1-k}{2}, \\
s & \text { if } s \geq \frac{1-k}{2} .\end{cases}
\end{aligned}
$$

Then $x y \in C_{(t, s)}(A)$ or $\mu_{A}(x y)+t+k>\frac{1-k}{2}+\frac{1-k}{2}+k=1$ and $\gamma_{A}(x y)+s+k<\frac{1-k}{2}+\frac{1-k}{2}+k=1$, that is, $x y \in Q_{(t, s)}^{k}(A)$. Hence $x y \in C_{(t, s)}(A) \cup Q_{(t, s)}^{k}(A)=[A]_{(t, s)}^{k}$. For the second case, assume that $t>\frac{1-k}{2}$ and $s<\frac{1-k}{2}$, then $1-t-k \leq 1-t<\frac{1-k}{2}$ and $1-s-k \geq 1-s \geq \frac{1-k}{2}$. Hence

$$
\begin{aligned}
\mu_{A}(x y) & \geq \min \left\{\mu_{A}(x), \mu_{A}(y), \frac{1-k}{2}\right\} \\
& = \begin{cases}\min \left\{\mu_{A}(y), \frac{1-k}{2}\right\}>1-t-k & \text { if } \min \left\{\mu_{A}(y), \frac{1-k}{2}\right\} \leq \mu_{A}(x), \\
\mu_{A}(x) \geq t & \text { if } \min \left\{\mu_{A}(y), \frac{1-k}{2}\right\}>\mu_{A}(x)\end{cases}
\end{aligned}
$$

and

$$
\begin{aligned}
\gamma_{A}(x y) & \leq \max \left\{\gamma_{A}(x), \gamma_{A}(y), \frac{1-k}{2}\right\} \\
& = \begin{cases}\max \left\{\gamma_{A}(y), \frac{1-k}{2}\right\}<1-s-k & \text { if } \max \left\{\gamma_{A}(y), \frac{1-k}{2}\right\} \geq \gamma_{A}(x), \\
\gamma_{A}(x) \leq s & \text { if } \max \left\{\gamma_{A}(y), \frac{1-k}{2}\right\}<\gamma_{A}(x) .\end{cases}
\end{aligned}
$$

Thus $x y \in C_{(t, s)}(A) \cup Q_{(t, s)}^{k}(A)=[A]_{(t, s)}^{k}$. Suppose that $t \leq \frac{1-k}{2}$ and $s \geq \frac{1-k}{2}$. Then

$$
\begin{aligned}
\mu_{A}(x y) & \geq \min \left\{\mu_{A}(x), \mu_{A}(y), \frac{1-k}{2}\right\} \\
& = \begin{cases}\min \left\{\mu_{A}(x), \frac{1-k}{2}\right\} \geq t & \text { if } \min \left\{\mu_{A}(x), \frac{1-k}{2}\right\} \leq \mu_{A}(y), \\
\mu_{A}(y)>1-t-k & \text { if } \min \left\{\mu_{A}(x), \frac{1-k}{2}\right\}>\mu_{A}(y),\end{cases}
\end{aligned}
$$

and

$$
\begin{aligned}
\gamma_{A}(x y) & \leq \max \left\{\gamma_{A}(x), \gamma_{A}(y), \frac{1-k}{2}\right\} \\
& = \begin{cases}\max \left\{\gamma_{A}(x), \frac{1-k}{2}\right\} \leq s & \text { if } \max \left\{\gamma_{A}(x), \frac{1-k}{2}\right\} \geq \gamma_{A}(y), \\
\gamma_{A}(y)<1-s-k & \text { if } \max \left\{\gamma_{A}(x), \frac{1-k}{2}\right\}<\gamma_{A}(y) .\end{cases}
\end{aligned}
$$


Thus $x y \in C_{(t, s)}(A) \cup Q_{(t, s)}^{k}(A)=[A]_{(t, s)}^{k}$. We have a similar result for the case (iii). For the final case, if $t>\frac{1-k}{2}$ and $s<\frac{1-k}{2}$, then $1-t-k<\frac{1-k}{2}$ and $1-s-k>\frac{1-k}{2}$. Hence

$$
\begin{aligned}
\mu_{A}(x y) & \geq \min \left\{\mu_{A}(x), \mu_{A}(y), \frac{1-k}{2}\right\} \\
& =\frac{1-k}{2}>1-t-k \quad \text { whenever } \min \left\{\mu_{A}(x), \mu_{A}(y)\right\} \geq \frac{1-k}{2}
\end{aligned}
$$

and

$$
\begin{aligned}
\mu_{A}(x y) & \leq \min \left\{\mu_{A}(x), \mu_{A}(y), \frac{1-k}{2}\right\} \\
& =\min \left\{\mu_{A}(x), \mu_{A}(y)\right\} \\
& >1-s-k \quad \text { whenever } \min \left\{\mu_{A}(x), \mu_{A}(y)\right\} \geq \frac{1-k}{2}
\end{aligned}
$$

and

$$
\begin{aligned}
\gamma_{A}(x y) & \leq \max \left\{\gamma_{A}(x), \gamma_{A}(y), \frac{1-k}{2}\right\} \\
& =\frac{1-k}{2}<1-s-k \text { whenever } \max \left\{\gamma_{A}(x), \gamma_{A}(y)\right\} \leq \frac{1-k}{2}
\end{aligned}
$$

and

$$
\begin{aligned}
\gamma_{A}(x y) & \leq \max \left\{\gamma_{A}(x), \gamma_{A}(y), \frac{1-k}{2}\right\} \\
& =\max \left\{\gamma_{A}(x), \gamma_{A}(y)\right\} \\
& <1-s-k, \quad \text { whenever } \max \left\{\gamma_{A}(x), \gamma_{A}(y)\right\} \leq \frac{1-k}{2} .
\end{aligned}
$$

Thus, $x y \in Q_{(t, s)}^{k}(A) \subseteq[A]_{(t, s)}^{k}$. If $t \leq \frac{1-k}{2}$ and $s \geq \frac{1-k}{2}$, then

$$
\begin{aligned}
\mu_{A}(x y) & \geq \min \left\{\mu_{A}(x), \mu_{A}(y), \frac{1-k}{2}\right\} \\
& = \begin{cases}\frac{1-k}{2} \geq t & \text { if } \min \left\{\mu_{A}(x), \mu_{A}(y)\right\} \geq \frac{1-k}{2}, \\
\min \left\{\mu_{A}(x), \mu_{A}(y)\right\}>1-t-k & \text { if } \min \left\{\mu_{A}(x), \mu_{A}(y)\right\}<\frac{1-k}{2},\end{cases}
\end{aligned}
$$

and

$$
\begin{aligned}
\gamma_{A}(x y) & \leq \max \left\{\gamma_{A}(x), \gamma_{A}(y), \frac{1-k}{2}\right\} \\
& = \begin{cases}\frac{1-k}{2} \leq s & \text { if } \max \left\{\gamma_{A}(x), \gamma_{A}(y)\right\} \leq \frac{1-k}{2}, \\
\max \left\{\gamma_{A}(x), \gamma_{A}(y)\right\}<1-s-k & \text { if } \max \left\{\gamma_{A}(x), \gamma_{A}(y)\right\}>\frac{1-k}{2},\end{cases}
\end{aligned}
$$

which implies that $x y \in Q_{(t, s)}^{k}(A) \subseteq[A]_{(t, s)}^{k}$. Let $x, z \in[A]_{(t, s)}^{k}$. Then $x \in C_{(t, s)}(A)$ or $\langle x$; $(t, s)\rangle \mathrm{q}_{k} A$ and $z \in C_{(t, s)}(A)$ or $\langle z ;(t, s)\rangle \mathrm{q}_{k} A$, that is, $\mu_{A}(x) \geq t, \gamma_{A}(x) \leq s$ or $\mu_{A}(x)+t+k>1$, 
$\gamma_{A}(x)+s+k<1$ and $\mu_{A}(z) \geq t, \gamma_{A}(z) \leq s$ or $\mu_{A}(z)+t+k>1, \gamma_{A}(z)+s+k<1$. We consider the following four cases

(i) $\mu_{A}(x) \geq t, \gamma_{A}(x) \leq s$ and $\mu_{A}(z) \geq t, \gamma_{A}(z) \leq s$,

(ii) $\mu_{A}(x) \geq t, \gamma_{A}(x) \leq s$ and $\mu_{A}(z)+t>1-k, \gamma_{A}(z)+s<1-k$,

(iii) $\mu_{A}(x)+t>1-k, \gamma_{A}(x)+s<1-k$ and $\mu_{A}(z) \geq t, \gamma_{A}(z) \leq s$,

(iv) $\mu_{A}(x)+t>1-k, \gamma_{A}(x)+s<1-k$ and $\mu_{A}(z)+t>1-k, \gamma_{A}(z)+s<1-k$.

For the case (i), Theorem 3.5(3) implies that

$$
\mu_{A}(x y z) \geq \min \left\{\mu_{A}(x), \mu_{A}(z), \frac{1-k}{2}\right\} \geq \min \left\{t, \frac{1-k}{2}\right\}= \begin{cases}\frac{1-k}{2} & \text { if } t>\frac{1-k}{2} \\ t & \text { if } t \leq \frac{1-k}{2}\end{cases}
$$

and

$$
\gamma_{A}(x y z) \leq \max \left\{\gamma_{A}(x), \gamma_{A}(z), \frac{1-k}{2}\right\} \leq \max \left\{s, \frac{1-k}{2}\right\}= \begin{cases}\frac{1-k}{2} & \text { if } s<\frac{1-k}{2} \\ s & \text { if } s \geq \frac{1-k}{2}\end{cases}
$$

Then $x y z \in C_{(t, s)}(A)$ or $\mu_{A}(x y z)+t+k>\frac{1-k}{2}+\frac{1-k}{2}+k=1$ and $\gamma_{A}(x y z)+s+k<\frac{1-k}{2}+\frac{1-k}{2}+$ $k=1$, that is, $x y z \in Q_{(t, s)}^{k}(A)$. Hence $x y z \in C_{(t, s)}(A) \cup Q_{(t, s)}^{k}(A)=[A]_{(t, s)}^{k}$. For the second case, assume that $t>\frac{1-k}{2}$ and $s<\frac{1-k}{2}$, then $1-t-k \leq 1-t<\frac{1-k}{2}$ and $1-s-k \geq 1-s \geq \frac{1-k}{2}$. Hence

$$
\begin{aligned}
\mu_{A}(x y z) & \geq \min \left\{\mu_{A}(x), \mu_{A}(z), \frac{1-k}{2}\right\} \\
& = \begin{cases}\min \left\{\mu_{A}(x), \frac{1-k}{2}\right\}>1-t-k & \text { if } \min \left\{\mu_{A}(z), \frac{1-k}{2}\right\} \leq \mu_{A}(x), \\
\mu_{A}(x) \geq t & \text { if } \min \left\{\mu_{A}(z), \frac{1-k}{2}\right\}>\mu_{A}(x)\end{cases}
\end{aligned}
$$

and

$$
\begin{aligned}
\gamma_{A}(x y z) & \leq \max \left\{\gamma_{A}(x), \gamma_{A}(z), \frac{1-k}{2}\right\} \\
& = \begin{cases}\max \left\{\gamma_{A}(z), \frac{1-k}{2}\right\}<1-s-k & \text { if } \max \left\{\gamma_{A}(z), \frac{1-k}{2}\right\} \geq \gamma_{A}(x), \\
\gamma_{A}(x) \leq s & \text { if } \max \left\{\gamma_{A}(z), \frac{1-k}{2}\right\}<\gamma_{A}(x) .\end{cases}
\end{aligned}
$$

Thus, $x y z \in C_{(t, s)}(A) \cup Q_{(t, s)}^{k}(A)=[A]_{(t, s)}^{k}$. Suppose that $t \leq \frac{1-k}{2}$ and $s \geq \frac{1-k}{2}$. Then

$$
\begin{aligned}
\mu_{A}(x y z) \geq & \min \left\{\mu_{A}(x), \mu_{A}(z), \frac{1-k}{2}\right\} \\
& = \begin{cases}\min \left\{\mu_{A}(x), \frac{1-k}{2}\right\} \geq t & \text { if } \min \left\{\mu_{A}(x), \frac{1-k}{2}\right\} \leq \mu_{A}(z), \\
\mu_{A}(z)>1-t-k & \text { if } \min \left\{\mu_{A}(x), \frac{1-k}{2}\right\}>\mu_{A}(z),\end{cases}
\end{aligned}
$$

and

$$
\begin{aligned}
\gamma_{A}(x y z) & \leq \max \left\{\gamma_{A}(x), \gamma_{A}(z), \frac{1-k}{2}\right\} \\
& = \begin{cases}\max \left\{\gamma_{A}(x), \frac{1-k}{2}\right\} \leq s & \text { if } \max \left\{\gamma_{A}(x), \frac{1-k}{2}\right\} \geq \gamma_{A}(z), \\
\gamma_{A}(y)<1-s-k & \text { if } \max \left\{\gamma_{A}(x), \frac{1-k}{2}\right\}<\gamma_{A}(z) .\end{cases}
\end{aligned}
$$


Thus, $x y z \in C_{(t, s)}(A) \cup Q_{(t, s)}^{k}(A)=[A]_{(t, s)}^{k}$. We have a similar result for the case (iii). For the final case, if $t>\frac{1-k}{2}$ and $s<\frac{1-k}{2}$, then $1-t-k<\frac{1-k}{2}$ and $1-s-k>\frac{1-k}{2}$. Hence

$$
\begin{aligned}
\mu_{A}(x y z) & \geq \min \left\{\mu_{A}(x), \mu_{A}(z), \frac{1-k}{2}\right\} \\
& =\frac{1-k}{2}>1-t-k \text { whenever } \min \left\{\mu_{A}(x), \mu_{A}(z)\right\} \geq \frac{1-k}{2}
\end{aligned}
$$

and

$$
\begin{aligned}
\mu_{A}(x y z) & \leq \min \left\{\mu_{A}(x), \mu_{A}(z), \frac{1-k}{2}\right\} \\
& =\min \left\{\mu_{A}(x), \mu_{A}(z)\right\} \\
& >1-s-k \quad \text { whenever } \min \left\{\mu_{A}(x), \mu_{A}(z)\right\} \geq \frac{1-k}{2}
\end{aligned}
$$

and

$$
\begin{aligned}
\gamma_{A}(x y z) & \leq \max \left\{\gamma_{A}(x), \gamma_{A}(z), \frac{1-k}{2}\right\} \\
& =\frac{1-k}{2}<1-s-k \text { whenever } \max \left\{\gamma_{A}(x), \gamma_{A}(z)\right\} \leq \frac{1-k}{2}
\end{aligned}
$$

and

$$
\begin{aligned}
\gamma_{A}(x y z) & \leq \max \left\{\gamma_{A}(x), \gamma_{A}(z), \frac{1-k}{2}\right\} \\
& =\max \left\{\gamma_{A}(x), \gamma_{A}(z)\right\} \\
& <1-s-k \text { whenever } \max \left\{\gamma_{A}(x), \gamma_{A}(z)\right\} \leq \frac{1-k}{2} .
\end{aligned}
$$

Thus, $x y z \in Q_{(t, s)}^{k}(A) \subseteq[A]_{(t, s)}^{k}$. If $t \leq \frac{1-k}{2}$ and $s \geq \frac{1-k}{2}$, then

$$
\begin{aligned}
\mu_{A}(x y z) & \geq \min \left\{\mu_{A}(x), \mu_{A}(z), \frac{1-k}{2}\right\} \\
& = \begin{cases}\frac{1-k}{2} \geq t & \text { if } \min \left\{\mu_{A}(x), \mu_{A}(z)\right\} \geq \frac{1-k}{2}, \\
\min \left\{\mu_{A}(x), \mu_{A}(z)\right\}>1-t-k & \text { if } \min \left\{\mu_{A}(x), \mu_{A}(z)\right\}<\frac{1-k}{2},\end{cases}
\end{aligned}
$$

and

$$
\begin{aligned}
\gamma_{A}(x y z) & \leq \max \left\{\gamma_{A}(x), \gamma_{A}(z), \frac{1-k}{2}\right\} \\
& = \begin{cases}\frac{1-k}{2} \leq s & \text { if } \max \left\{\gamma_{A}(x), \gamma_{A}(z)\right\} \leq \frac{1-k}{2}, \\
\max \left\{\gamma_{A}(x), \gamma_{A}(z)\right\}<1-s-k & \text { if } \max \left\{\gamma_{A}(x), \gamma_{A}(z)\right\}>\frac{1-k}{2},\end{cases}
\end{aligned}
$$

which implies that $x y z \in Q_{(t, s)}^{k}(A) \subseteq[A]_{(t, s)}^{k}$. Therefore, $[A]_{(t, s)}^{k}$ is a bi-ideal of $S$. 
Conversely, suppose that (2) is valid. If there exist $a, b \in S$ such that $a \leq b$ and

$$
\mu_{A}(a)<\min \left\{\mu_{A}(b), \frac{1-k}{2}\right\} \quad \text { and } \quad \gamma_{A}(a)>\max \left\{\gamma_{A}(b), \frac{1-k}{2}\right\}
$$

Then $\mu_{A}(a)<t_{a} \leq \min \left\{\mu_{A}(b), \frac{1-k}{2}\right\}$ and $\gamma_{A}(a)>s_{a} \geq \max \left\{\gamma_{A}(b), \frac{1-k}{2}\right\}$ for some $t_{a} \in(0,1]$ and $s_{a} \in[0,1)$. It follows that $b \in C_{\left(t_{a}, s_{a}\right)}(A) \subseteq[A]_{\left(t_{a}, s_{a}\right)}^{k}$ but $a \notin C_{\left(t_{a}, s_{a}\right)}(A)$. Also we have $\mu_{A}(a)+t_{a}<2 t_{a} \leq 1-k$ and $\gamma_{A}(a)+s_{a}>2 s_{a} \geq 1-k$ and so $\left\langle a ;\left(t_{a}, s_{a}\right)\right\rangle \overline{\mathrm{q}_{k}} A$, i.e., $b \notin$ $Q_{\left(t_{a}, s_{a}\right)}^{k}(A)$. Therefore, $a \notin[A]_{\left(t_{a}, s_{a}\right)}$, a contradiction. Hence $\mu_{A}(x) \geq \min \left\{\mu_{A}(y), \frac{1-k}{2}\right\}$ and $\gamma_{A}(x) \leq \max \left\{\gamma_{A}(y), \frac{1-k}{2}\right\}$ for all $x, y \in S$ with $x \leq y$. Suppose that there exist $a, b \in S$ such that

$$
\mu_{A}(a b)<\min \left\{\mu_{A}(a), \mu_{A}(b), \frac{1-k}{2}\right\}
$$

and

$$
\gamma_{A}(a b)>\max \left\{\gamma_{A}(a), \gamma_{A}(b), \frac{1-k}{2}\right\}
$$

Then

$$
\mu_{A}(a b)<t \leq \min \left\{\mu_{A}(a), \mu_{A}(b), \frac{1-k}{2}\right\}
$$

and

$$
\gamma_{A}(a b)>s \geq \max \left\{\gamma_{A}(a), \gamma_{A}(b), \frac{1-k}{2}\right\}
$$

for $t \in(0,1]$ and $s \in[0,1)$. It follows that $a \in C_{(t, s)}(A) \subseteq[A]_{(t, s)}^{k}$ and $b \in C_{(t, s)}(A) \subseteq$ $[A]_{(t, s)}^{k}$, so from (b2) $a b \in[A]_{(t, s)}^{k}$. Thus, $\mu_{A}(a b) \geq t, \gamma_{A}(a b) \leq s$ or $\mu_{A}(a b)+t+k>1$, $\gamma_{A}(a b)+s+k<1$, a contradiction. Therefore, $\mu_{A}(x y) \geq \min \left\{\mu_{A}(x), \mu_{A}(y), \frac{1-k}{2}\right\}$ and $\gamma_{A}(x y) \leq$ $\max \left\{\gamma_{A}(x), \gamma_{A}(y), \frac{1-k}{2}\right\}$ for all $x, y \in S$. Assume that there exist $a, b, c \in S$ such that

$$
\mu_{A}(a b c)<\min \left\{\mu_{A}(a), \mu_{A}(c), \frac{1-k}{2}\right\}
$$

and

$$
\gamma_{A}(a b c)>\max \left\{\gamma_{A}(a), \gamma_{A}(c), \frac{1-k}{2}\right\}
$$

Then $\mu_{A}(a b c)<t_{0} \leq \min \left\{\mu_{A}(a), \mu_{A}(c), \frac{1-k}{2}\right\}$ and $\gamma_{A}(a b c)>s_{0} \geq \max \left\{\gamma_{A}(a), \gamma_{A}(c), \frac{1-k}{2}\right\}$ for $t_{0} \in(0,1]$ and $s_{0} \in[0,1)$. It follows that $a \in C_{\left(t_{0}, s_{0}\right)}(A) \subseteq[A]_{\left(t_{0}, s_{0}\right)}^{k}$ and $c \in C_{\left(t_{0}, s_{0}\right)}(A) \subseteq$ $[A]_{\left(t_{0}, s_{0}\right)}^{k}$ so from (b2) $a b c \in[A]_{\left(t_{0}, s_{0}\right)}$. Thus, $\mu_{A}(a b) \geq t_{0}, \gamma_{A}(a b) \leq s_{0}$ or $\mu_{A}(a b)+t_{0}+k>$ $1, \gamma_{A}(a b)+s_{0}+k<1$, a contradiction. Therefore, $\mu_{A}(x y z) \geq \min \left\{\mu_{A}(x), \mu_{A}(z), \frac{1-k}{2}\right\}$ and $\gamma_{A}(x y z) \leq \max \left\{\gamma_{A}(x), \gamma_{A}(z), \frac{1-k}{2}\right\}$ for all $x, y, z \in S$. Thus, $A=\left\langle x, \mu_{A}, \gamma_{A}\right\rangle$ is an $\left(\in, \in \vee \mathrm{q}_{k}\right)$ intuitionistic fuzzy bi-ideal of $S$. 
Theorem 3.14 Let $\left\{A_{i} \mid i \in \Lambda\right\}$ be a family of $\left(\in, \in \vee \mathrm{q}_{k}\right)$-intuitionistic fuzzy bi-ideals of $S$. Then $A=\bigcap_{i \in \Lambda} A_{i}$ is an $\left(\in, \in \vee \mathrm{q}_{k}\right)$-intuitionistic fuzzy bi-ideal of $S$, where $\bigcap_{i \in \Lambda} A_{i}=$ $\left\langle x, \bigwedge_{i \in \Lambda} \mu_{A_{i}}, \bigvee_{i \in \Lambda} \gamma_{A i}\right\rangle$ and

$$
\begin{aligned}
& \bigwedge_{i \in \Lambda} \mu_{A_{i}}(x)=\inf \left\{\mu_{A_{i}}(x) \mid i \in \Lambda \text { and } x \in S\right\} \\
& \bigvee_{i \in \Lambda} \gamma_{A_{i}}(x)=\sup \left\{\gamma_{A_{i}}(x) \mid i \in \Lambda \text { and } x \in S\right\} .
\end{aligned}
$$

Proof Let $x, y \in S$ with $x \leq y, t \in(0,1]$ and $s \in[0,1)$ be such that $\langle y ;(t, s)\rangle \in A$. Assume that $\langle x ;(t, s)\rangle \overline{\epsilon \vee \mathrm{q}_{k}} A$. Then $\mu_{A}(x)<t, \gamma_{A}(x)>s$ and $\mu_{A}(x)+t+k \leq 1, \gamma_{A}(x)+s+k \geq 1$, which imply that

$$
\mu_{A}(x)<\frac{1-k}{2} \text { and } \quad \gamma_{A}(x)>\frac{1-k}{2} .
$$

Let $\Omega_{1}:=\left\{i \in \Lambda \mid \mu_{A_{i}}(x) \geq t, \gamma_{A_{i}}(x) \leq s\right\}$ and $\Omega_{2}:=\left\{i \in \Lambda \mid\langle x ;(t, s)\rangle \mathrm{q}_{k} A_{i}\right.$ and $\mu_{A_{i}}(x)<$ $\left.t, \gamma_{A_{i}}(x)>s\right\}$.

Then $\Lambda=\Omega_{1} \cup \Omega_{2}$ and $\Omega_{1} \cap \Omega_{2}=\emptyset$. If $\Omega_{2}=\emptyset$, then $\mu_{A_{i}}(x) \geq t, \gamma_{A_{i}}(x) \leq s$ for all $i \in \Lambda$, and so, $\mu_{A}(x) \geq t, \gamma_{A}(x) \leq s$, which is a contradiction. Hence $\Omega_{2} \neq \emptyset$, and so, $\mu_{A_{i}}(x)+t+k>1$, $\gamma_{A_{i}}(x)+s+k<1$ and $\mu_{A_{i}}(x)<t, \gamma_{A_{i}}(x)>s$ for every $i \in \Lambda$. It follows that $t>\frac{1-k}{2}$ and $s<\frac{1-k}{2}$, so that $\mu_{A_{i}}(x) \geq \mu_{A}(x) \geq t>\frac{1-k}{2}$ and $\gamma_{A_{i}}(x) \leq \gamma_{A}(x) \leq s<\frac{1-k}{2}$ for all $i \in \Lambda$. Now, suppose that $t_{x}:=\mu_{A_{i}}(x)<\frac{1-k}{2}$ and $s_{x}:=\gamma_{A_{i}}(x)>\frac{1-k}{2}$ for some $i \in \Lambda$. Let $t_{x}^{\prime} \in\left(0, \frac{1-k}{2}\right)$ and $s_{x}^{\prime} \in\left(\frac{1-k}{2}, 1\right)$ be such that $t_{x}<t_{x}^{\prime}$ and $s_{x}^{\prime}<s_{x}$. Then $\mu_{A_{i}}(y)>\frac{1-k}{2}>t_{x}^{\prime}$ and $\gamma_{A_{i}}(y)<\frac{1-k}{2}<s_{x}^{\prime}$, i.e., $\left\langle y ;\left(t_{x}^{\prime}, s_{x}^{\prime}\right)\right\rangle \in$ $A_{i}$. But $\mu_{A_{i}}(x)=t_{x}<t_{x}^{\prime}, \gamma_{A_{i}}(x)=s_{x}>s_{x}^{\prime}$ and $\mu_{A_{i}}(x)+t_{x}^{\prime}+k<1, \gamma_{A_{i}}(x)+s_{x}^{\prime}+k>1$, that is, $\left\langle x ;\left(t_{x}^{\prime}, s_{x}^{\prime}\right)\right\rangle \overline{\in \vee \mathrm{q}_{k}} A_{i}$. This is a contradiction, and so, $\mu_{A_{i}}(x) \geq \frac{1-k}{2}$ and $\gamma_{A_{i}}(x) \leq \frac{1-k}{2}$ for all $i \in \Lambda$. Thus, $\mu_{A}(x) \geq \frac{1-k}{2}$ and $\gamma_{A}(x) \leq \frac{1-k}{2}$, which is impossible. Therefore, $\langle y ;(t, s)\rangle \in \vee \mathrm{q}_{k} A$.

Let $x, y \in S, t_{1}, t_{2} \in(0,1]$ and $s_{1}, s_{2} \in[0,1)$ be such that $\left\langle x ;\left(t_{1}, s_{1}\right)\right\rangle \in A$ and $\left\langle y ;\left(t_{2}, s_{2}\right)\right\rangle \in A$. Assume that $\left\langle x y ; \min \left\{t_{1}, t_{2}\right\}, \max \left\{s_{1}, s_{2}\right\}\right\rangle \overline{\in \vee \mathrm{q}_{k}} A$. Then

$$
\mu_{A}(x y)<\min \left\{t_{1}, t_{2}\right\}, \quad \gamma_{A}(x y)>\max \left\{s_{1}, s_{2}\right\}
$$

and

$$
\mu_{A}(x y)+\min \left\{t_{1}, t_{2}\right\}<1-k, \quad \gamma_{A}(x y)+\max \left\{s_{1}, s_{2}\right\}>1-k .
$$

It follows that $\mu_{A}(x y)<\frac{1-k}{2}$ and $\gamma_{A}(x y)>\frac{1-k}{2}$. Let $\Omega_{3}:=\left\{i \in \Lambda \mid \mu_{A_{i}}(x y) \geq \min \left\{t_{1}, t_{2}\right\}\right.$ and $\left.\gamma_{A_{i}}(x y) \leq \max \left\{s_{1}, s_{2}\right\}\right\}$ and $\Omega_{4}:=\left\{i \in \Lambda \mid\left\langle x y ; \min \left\{t_{1}, t_{2}\right\}, \max \left\{s_{1}, s_{2}\right\}\right\rangle \mathrm{q}_{k} A_{i}\right.$ and $\mu_{A_{i}}(x y)<$ $\min \left\{t_{1}, t_{2}\right\}$ and $\left.\gamma_{A_{i}}(x y)>\max \left\{s_{1}, s_{2}\right\}\right\}$. Then $\Omega_{3} \cup \Omega_{4}=\Lambda$ and $\Omega_{3} \cap \Omega_{4}=\emptyset$. If $\Omega_{4}=\emptyset$, then $\mu_{A_{i}}(x y) \geq \min \left\{t_{1}, t_{2}\right\}$ and $\gamma_{A_{i}}(x y) \leq \max \left\{s_{1}, s_{2}\right\}$ for all $i \in \Lambda$, and so, $\mu_{A}(x y) \geq \min \left\{t_{1}, t_{2}\right\}$ and $\gamma_{A}(x y) \leq \max \left\{s_{1}, s_{2}\right\}$, which is a contradiction. Hence $\Omega_{4} \neq \emptyset$ and $\left\langle x y ; \min \left\{t_{1}, t_{2}\right\}, \max \left\{s_{1}\right.\right.$, $\left.\left.s_{2}\right\}\right\rangle \mathrm{q}_{k} A_{i}$, i.e., $\mu_{A_{i}}(x y)+\min \left\{t_{1}, t_{2}\right\}>1-k, \gamma_{A_{i}}(x y)+\max \left\{s_{1}, s_{2}\right\}<1-k$. It follows that $\min \left\{t_{1}, t_{2}\right\}>\frac{1-k}{2}$ and $\max \left\{s_{1}, s_{2}\right\}<\frac{1-k}{2}$, so that $\mu_{A_{i}}(x) \geq \mu_{A}(x) \geq t_{1} \geq \min \left\{t_{1}, t_{2}\right\}>\frac{1-k}{2}$ and $\gamma_{A_{i}}(x) \leq \gamma_{A}(x) \leq s_{1} \leq \max \left\{s_{1}, s_{2}\right\}$ for all $i \in \Lambda$. By a similar way, we have $\mu_{A_{i}}(y) \geq \mu_{A}(y) \geq$ $t_{1} \geq \min \left\{t_{1}, t_{2}\right\}>\frac{1-k}{2}$ and $\gamma_{A_{i}}(y) \leq \gamma_{A}(y) \leq s_{1} \leq \max \left\{s_{1}, s_{2}\right\}$ for all $i \in \Lambda$. Now, suppose that $t:=\mu_{A_{i}}(x y)<\frac{1-k}{2}$ and $s:=\gamma_{A_{i}}(x y)>\frac{1-k}{2}$ for some $i \in \Lambda$. Let $t^{\prime} \in\left(0, \frac{1-k}{2}\right)$ and $s^{\prime} \in\left(\frac{1-k}{2}, 1\right)$ be such that $t<t^{\prime}$ and $s>s^{\prime}$. Then $\mu_{A_{i}}(x)>\frac{1-k}{2}>t^{\prime}, \gamma_{A_{i}}(x)<\frac{1-k}{2}<s^{\prime}$ and $\mu_{A_{i}}(y)>\frac{1-k}{2}>t^{\prime}$, 
$\gamma_{A_{i}}(y)<\frac{1-k}{2}<s^{\prime}$, i.e., $\left\langle x ;\left(t^{\prime}, s^{\prime}\right)\right\rangle \in A$ and $\left\langle y ;\left(t^{\prime}, s^{\prime}\right)\right\rangle \in A$. But $\mu_{A_{i}}(x y)=t<t^{\prime}, \gamma_{A_{i}}(x y)=s>s^{\prime}$ and $\mu_{A_{i}}(x y)+t^{\prime}+k<1, \gamma_{A_{i}}(x y)+s^{\prime}+k>1$, that is, $\left\langle x y ;\left(t^{\prime}, s^{\prime}\right)\right\rangle \bar{\epsilon} \vee \overline{\mathrm{q}_{k}} A_{i}$. This is a contradiction. Thus, $\mu_{A_{i}}(x y) \geq \frac{1-k}{2}$ and $\gamma_{A_{i}}(x y) \leq \frac{1-k}{2}$ for all $i \in \Lambda$. Therefore, $\mu_{A}(x y) \geq \frac{1-k}{2}$ and $\gamma_{A}(x y) \leq \frac{1-k}{2}$, which is invalid. Consequently, $\left\langle x y ; \min \left\{t_{1}, t_{2}\right\}, \max \left\{s_{1}, s_{2}\right\}\right\rangle \in \vee \mathrm{q}_{k} A$. Finally, suppose that $x, y, z \in S, t_{1}, t_{2} \in(0,1]$ and $s_{1}, s_{2} \in[0,1)$ be such that $\left\langle x ;\left(t_{1}, s_{1}\right)\right\rangle \in A$ and $\left\langle z ;\left(t_{2}, s_{2}\right)\right\rangle \in A$. Assume that $\left\langle x y z ; \min \left\{t_{1}, t_{2}\right\}, \max \left\{s_{1}, s_{2}\right\}\right\rangle \overline{\in \vee \mathrm{q}_{k}} A$. Then

$$
\mu_{A}(x y z)<\min \left\{t_{1}, t_{2}\right\}, \quad \gamma_{A}(x y z)>\max \left\{s_{1}, s_{2}\right\}
$$

and

$$
\mu_{A}(x y z)+\min \left\{t_{1}, t_{2}\right\}<1-k, \quad \gamma_{A}(x y z)+\max \left\{s_{1}, s_{2}\right\}>1-k .
$$

It follows that $\mu_{A}(x y z)<\frac{1-k}{2}$ and $\gamma_{A}(x y z)>\frac{1-k}{2}$. Let

$$
\Omega_{5}:=\left\{i \in \Lambda \mid \mu_{A_{i}}(x y z) \geq \min \left\{t_{1}, t_{2}\right\} \text { and } \gamma_{A_{i}}(x y z) \leq \max \left\{s_{1}, s_{2}\right\}\right\}
$$

and

$$
\begin{aligned}
\Omega_{6}:= & \left\{i \in \Lambda|| x y z ; \min \left\{t_{1}, t_{2}\right\}, \max \left\{s_{1}, s_{2}\right\}\right\rangle \mathrm{q}_{k} A_{i} \\
& \text { and } \left.\mu_{A_{i}}(x y z)<\min \left\{t_{1}, t_{2}\right\} \text { and } \gamma_{A_{i}}(x y z)>\max \left\{s_{1}, s_{2}\right\}\right\} .
\end{aligned}
$$

Then $\Omega_{5} \cup \Omega_{6}=\Lambda$ and $\Omega_{5} \cap \Omega_{6}=\emptyset$. If $\Omega_{6}=\emptyset$, then $\mu_{A_{i}}(x y z) \geq \min \left\{t_{1}, t_{2}\right\}$ and $\gamma_{A_{i}}(x y z) \leq$ $\max \left\{s_{1}, s_{2}\right\}$ for all $i \in \Lambda$, and so $\mu_{A}(x y z) \geq \min \left\{t_{1}, t_{2}\right\}$ and $\gamma_{A}(x y z) \leq \max \left\{s_{1}, s_{2}\right\}$ which is a contradiction. Hence $\Omega_{6} \neq \emptyset$ and

$$
\left\langle x y z ; \min \left\{t_{1}, t_{2}\right\}, \max \left\{s_{1}, s_{2}\right\}\right\rangle \mathrm{q}_{k} A_{i},
$$

i.e.,

$$
\mu_{A_{i}}(x y z)+\min \left\{t_{1}, t_{2}\right\}>1-k, \quad \gamma_{A_{i}}(x y z)+\max \left\{s_{1}, s_{2}\right\}<1-k .
$$

It follows that $\min \left\{t_{1}, t_{2}\right\}>\frac{1-k}{2}$ and $\max \left\{s_{1}, s_{2}\right\}<\frac{1-k}{2}$, so that

$$
\mu_{A_{i}}(x) \geq \mu_{A}(x) \geq t_{1} \geq \min \left\{t_{1}, t_{2}\right\}>\frac{1-k}{2}
$$

and

$$
\gamma_{A_{i}}(x) \leq \gamma_{A}(x) \leq s_{1} \leq \max \left\{s_{1}, s_{2}\right\}
$$

for all $i \in \Lambda$. Similarly, we have

$$
\mu_{A_{i}}(z) \geq \mu_{A}(z) \geq t_{1} \geq \min \left\{t_{1}, t_{2}\right\}>\frac{1-k}{2}
$$

and

$$
\gamma_{A_{i}}(z) \leq \gamma_{A}(z) \leq s_{1} \leq \max \left\{s_{1}, s_{2}\right\}
$$


for all $i \in \Lambda$. Now, suppose that $t:=\mu_{A_{i}}(x y z)<\frac{1-k}{2}$ and $s:=\gamma_{A_{i}}(x y z)>\frac{1-k}{2}$ for some $i \in \Lambda$. Let $t^{\prime} \in\left(0, \frac{1-k}{2}\right)$ and $s^{\prime} \in\left(\frac{1-k}{2}, 1\right)$ be such that $t<t^{\prime}$ and $s>s^{\prime}$. Then $\mu_{A_{i}}(x)>\frac{1-k}{2}>t^{\prime}, \gamma_{A_{i}}(x)<$ $\frac{1-k}{2}<s^{\prime}$ and $\mu_{A_{i}}(y)>\frac{1-k}{2}>t^{\prime}, \gamma_{A_{i}}(y)<\frac{1-k}{2}<s^{\prime}$, i.e., $\left\langle x ;\left(t^{\prime}, s^{\prime}\right)\right\rangle \in A$ and $\left\langle y ;\left(t^{\prime}, s^{\prime}\right)\right\rangle \in A$. But

$$
\mu_{A_{i}}(x y z)=t<t^{\prime}, \quad \gamma_{A_{i}}(x y z)=s>s^{\prime}
$$

and

$$
\mu_{A_{i}}(x y z)+t^{\prime}<1, \quad \gamma_{A_{i}}(x y z)+s^{\prime}>1
$$

that is, $\left\langle x y z ;\left(t^{\prime}, s^{\prime}\right)\right\rangle \bar{\in} \vee \overline{\mathrm{q}_{k}} A_{i}$. This is a contradiction. Thus, $\mu_{A_{i}}(x y z) \geq \frac{1-k}{2}$ and $\gamma_{A_{i}}(x y z) \leq$ $\frac{1-k}{2}$ for all $i \in \Lambda$. Therefore, $\mu_{A}(x y z) \geq \frac{1-k}{2}$ and $\gamma_{A}(x y z) \leq \frac{1-k}{2}$, which is invalid. Thus, $\left\langle x y z ; \min \left\{t_{1}, t_{2}\right\}, \max \left\{s_{1}, s_{2}\right\}\right\rangle \in \vee \mathrm{q}_{k} A$. Therefore, $\bigcap_{i \in \Lambda} A_{i}$ is an $\left(\in, \in \vee \mathrm{q}_{k}\right)$-intuitionistic fuzzy bi-ideal of $S$.

The following example shows that the union of two $\left(\in, \in \vee \mathrm{q}_{k}\right)$-intuitionistic fuzzy biideals of $S$ may not be an $\left(\in, \in \vee \mathrm{q}_{k}\right)$-intuitionistic fuzzy bi-ideal of $S$.

Example 3.15 Consider the ordered semigroup of Example 3.4 with the $*$-multiplication Table 1 and the IFS of Examples 3.4 and 3.7, then $\langle a ;(0.7,0.3)\rangle \in A \cup B$ and $\langle b ;(0.5,0.4)\rangle \in$ $A \cup B$, but $\langle a b \min \{0.7,0.5\}, \max \{0.3,0.4\}\rangle=\langle d ;(0.5,0.4)\rangle \overline{\in \vee \mathrm{q}_{k}} A \cup B$.

Definition 3.16 An IFS $A=\left\langle x, \mu_{A}, \gamma_{A}\right\rangle$ of $S$ is called an $\left(\bar{\epsilon}, \bar{\epsilon} \vee \overline{\mathrm{q}_{k}}\right)$-intuitionistic fuzzy biideal of $S$ if for all $x, y, z \in S, t, t_{1}, t_{2} \in(0,1]$ and $s, s_{1}, s_{2} \in[0,1)$, it satisfies the following conditions

(q4) $\left(\langle x ;(t, s)\rangle \bar{\epsilon} A \Longrightarrow\langle y ;(t, s)\rangle \bar{\epsilon} \vee \overline{\mathrm{q}_{k}} A\right.$ with $\left.x \leq y\right)$,

(q5) $\left(\left\langle x y ; \min \left\{t_{1}, t_{2}\right\}, \max \left\{s_{1}, s_{2}\right\}\right\rangle \bar{\epsilon} A \Longrightarrow\left\langle x ;\left(t_{1}, s_{1}\right)\right\rangle \bar{\epsilon} \vee \overline{\mathrm{q}_{k}} A\right.$ or $\left.\left\langle y ;\left(t_{2}, s_{2}\right)\right\rangle \bar{\epsilon} \vee \overline{\mathrm{q}_{k}} A\right)$,

(q6) $\left(\left\langle x y z ; \min \left\{t_{1}, t_{2}\right\}, \max \left\{s_{1}, s_{2}\right\}\right\rangle \bar{\epsilon} A \Longrightarrow\left\langle x ;\left(t_{1}, s_{1}\right)\right\rangle \bar{\in} \vee \overline{\mathrm{q}_{k}} A\right.$ or $\left.\left\langle z ;\left(t_{2}, s_{2}\right)\right\rangle \bar{\epsilon} \vee \overline{\mathrm{q}_{k}} A\right)$.

Let $A=\left\langle x, \mu_{A}, \gamma_{A}\right\rangle$ be an $\left(\bar{\epsilon}, \bar{\epsilon} \vee \overline{\mathrm{q}_{k}}\right)$-intuitionistic fuzzy bi-ideal of an ordered semigroup $S$. Suppose that there exist $a, b \in S$ with $a \leq b$ such that

$$
\mu_{A}(b)>\max \left\{\mu_{A}(a), \frac{1-k}{2}\right\} \quad \text { and } \quad \gamma_{A}(b)<\min \left\{\gamma_{A}(a), \frac{1-k}{2}\right\} \text {. }
$$

Then $\mu_{A}(b) \geq t>\max \left\{\mu_{A}(a), \frac{1-k}{2}\right\}$ and $\gamma_{A}(b) \leq s<\min \left\{\gamma_{A}(a), \frac{1-k}{2}\right\}$ for some $t \in\left(\frac{1-k}{2}, 1\right]$ and $s \in\left[0, \frac{1-k}{2}\right)$. It follows that $\langle a ;(t, s)\rangle \bar{\epsilon} A,\langle b ;(t, s)\rangle \in A$ and $\mu_{A}(b)+t \geq 2 t>1-k, \gamma_{A}(b)+$ $s \leq 2 s<1-k$, i.e., $\langle b ;(t, s)\rangle \mathrm{q}_{k} A$. This is a contradiction, and so the following inequalities hold.

$$
\text { (e1) }\left(x \leq y \Longrightarrow \mu_{A}(b) \leq \max \left\{\mu_{A}(a), \frac{1-k}{2}\right\} \text { and } \gamma_{A}(b) \geq \min \left\{\gamma_{A}(a), \frac{1-k}{2}\right\}\right) \text {. }
$$

Suppose that $\max \left\{\mu_{A}(a b), \frac{1-k}{2}\right\}<\min \left\{\mu_{A}(a), \mu_{A}(b)\right\}$ and $\min \left\{\gamma_{A}(a b), \frac{1-k}{2}\right\}>\max \left\{\gamma_{A}(a)\right.$, $\left.\gamma_{A}(b)\right\}$ for some $a, b \in S$. Then $\max \left\{\mu_{A}(a b), \frac{1-k}{2}\right\}<t \leq \min \left\{\mu_{A}(a), \mu_{A}(b)\right\}$ and $\min \left\{\gamma_{A}(a b)\right.$, $\left.\frac{1-k}{2}\right\}>s \geq \max \left\{\gamma_{A}(a), \gamma_{A}(b)\right\}$. Thus, $\langle a ;(t, s)\rangle \bar{\epsilon} A,\langle b ;(t, s)\rangle \bar{\epsilon} A, \mu_{A}(a)+t \geq 2 t>1-k$, $\gamma_{A}(a)+s \leq 2 s<1-k$, i.e., $\langle a ;(t, s)\rangle \mathrm{q}_{k} A$ and $\mu_{A}(b)+t \geq 2 t>1-k, \gamma_{A}(b)+s \leq 2 s<1-k$, i.e., $\langle b ;(t, s)\rangle \mathrm{q}_{k} A$. This is impossible, and hence $A=\left\langle x, \mu_{A}, \gamma_{A}\right\rangle$ satisfies the following assertion 
(e2) $\max \left\{\mu_{A}(x y), \frac{1-k}{2}\right\}<\min \left\{\mu_{A}(x), \mu_{A}(y)\right\}$ and $\min \left\{\gamma_{A}(x y), \frac{1-k}{2}\right\}>\max \left\{\gamma_{A}(x), \gamma_{A}(y)\right\}$ for all $x, y \in S$.

Now, assume that

$$
\max \left\{\mu_{A}(a b c), \frac{1-k}{2}\right\}<\min \left\{\mu_{A}(a), \mu_{A}(c)\right\}
$$

and

$$
\min \left\{\gamma_{A}(a b c), \frac{1-k}{2}\right\}>\max \left\{\gamma_{A}(a), \gamma_{A}(c)\right\}
$$

for some $a, b, c \in S$. Then

$$
\max \left\{\mu_{A}(a b c), \frac{1-k}{2}\right\}<t \leq \min \left\{\mu_{A}(a), \mu_{A}(c)\right\}
$$

and

$$
\min \left\{\gamma_{A}(a b c), \frac{1-k}{2}\right\}>s \geq \max \left\{\gamma_{A}(a), \gamma_{A}(c)\right\}
$$

Thus, $\langle a ;(t, s)\rangle \bar{\epsilon} A,\langle c ;(t, s)\rangle \bar{\epsilon} A, \mu_{A}(a)+t \geq 2 t>1-k, \gamma_{A}(a)+s \leq 2 s<1-k$, i.e., $\langle a ;(t, s)\rangle \mathrm{q}_{k} A$ and $\mu_{A}(c)+t \geq 2 t>1-k, \gamma_{A}(c)+s \leq 2 s<1-k$, i.e., $\langle c ;(t, s)\rangle \mathrm{q}_{k} A$. This is a contradiction, and hence we have the following assertion

(e3) $\max \left\{\mu_{A}(x y z), \frac{1-k}{2}\right\}<\min \left\{\mu_{A}(x), \mu_{A}(z)\right\}$ and $\min \left\{\gamma_{A}(x y z), \frac{1-k}{2}\right\}>\max \left\{\gamma_{A}(x), \gamma_{A}(z)\right\}$ for all $x, y, z \in S$.

Let $A=\left\langle x, \mu_{A}, \gamma_{A}\right\rangle$ be an IFS of $S$ satisfying the three conditions (e1), (e2) and (e3). Let $t \in\left(\frac{1-k}{2}, 1\right]$ and $s \in\left[0, \frac{1-k}{2}\right)$ be such that $C_{(t, s)}(A) \neq \emptyset$. Then there exist $b \in C_{(t, s)}(A)$ and $S \ni$ $a \leq b$, by using (e1), we get

$$
\frac{1-k}{2}<t \leq \mu_{A}(b) \leq \max \left\{\mu_{A}(a), \frac{1-k}{2}\right\}=\mu_{A}(a)
$$

and

$$
\frac{1-k}{2}>s \geq \gamma_{A}(b) \geq \min \left\{\gamma_{A}(a), \frac{1-k}{2}\right\}=\gamma_{A}(a) .
$$

Hence $a \in C_{(t, s)}(A)$. Let $a, b \in S$ be such that $a \in C_{(t, s)}(A)$ and $b \in C_{(t, s)}(A)$. Then $\mu_{A}(a) \geq t$, $\gamma_{A}(a) \leq s$ and $\mu_{A}(b) \geq t, \gamma_{A}(b) \leq s$. Using (e2), we get

$$
\max \left\{\mu_{A}(a b), \frac{1-k}{2}\right\} \geq \min \left\{\mu_{A}(a), \mu_{A}(b)\right\} \geq t>\frac{1-k}{2}
$$

and

$$
\min \left\{\gamma_{A}(a b), \frac{1-k}{2}\right\} \leq \max \left\{\gamma_{A}(a), \gamma_{A}(b)\right\} \leq s<\frac{1-k}{2},
$$

which implies that $\mu_{A}(a b)=\max \left\{\mu_{A}(a b), \frac{1-k}{2}\right\} \geq t$ and $\gamma_{A}(a b)=\min \left\{\gamma_{A}(a b), \frac{1-k}{2}\right\} \leq s$. Thus, $a b \in C_{(t, s)}(A)$. Now, suppose that $a, c \in C_{(t, s)}(A)$ and $b \in S$. Then $\mu_{A}(a) \geq t, \gamma_{A}(a) \leq s$ and 
$\mu_{A}(c) \geq t, \gamma_{A}(c) \leq s$. Using (e3), we get

$$
\max \left\{\mu_{A}(a b c), \frac{1-k}{2}\right\} \geq \min \left\{\mu_{A}(a), \mu_{A}(c)\right\} \geq t>\frac{1-k}{2}
$$

and

$$
\min \left\{\gamma_{A}(a b c), \frac{1-k}{2}\right\} \leq \max \left\{\gamma_{A}(a), \gamma_{A}(c)\right\} \leq s<\frac{1-k}{2}
$$

which implies that $\mu_{A}(a b c)=\max \left\{\mu_{A}(a b c), \frac{1-k}{2}\right\} \geq t$ and $\gamma_{A}(a b c)=\min \left\{\gamma_{A}(a b c), \frac{1-k}{2}\right\} \leq s$. Thus, $a b c \in C_{(t, s)}(A)$. Consequently, $C_{(t, s)}(A)$ is a bi-ideal of $S$. Therefore, we conclude that if an IFS $A=\left\langle x, \mu_{A}, \gamma_{A}\right\rangle$ of $S$ satisfies the three conditions (e1), (e2) and (e3), then the following assertion is valid

(e4) $(\forall t \in(0.5,1])\left(\forall s \in\left[0, \frac{1-k}{2}\right)\right)\left(C_{(t, s)}(A) \neq \emptyset \Longrightarrow C_{(t, s)}(A)\right.$ is a bi-ideal of $\left.S\right)$.

Now, let $A=\left\langle x, \mu_{A}, \gamma_{A}\right\rangle$ be an IFS of S satisfying (e4). Let $a, b \in S$ with $a \leq b$ and $t \in(0,1]$ and $s \in[0,1)$ be such that $\langle b ;(t, s)\rangle \overline{\bar{\epsilon} \vee \overline{\mathrm{q}_{k}}} A$. Then $\langle b ;(t, s)\rangle \in A$ and $\langle b ;(t, s)\rangle \mathrm{q}_{k} A$. Hence $b \in C_{(t, s)}(A)$ and $C_{(t, s)}(A) \neq \emptyset$. Thus, by (e4), $a \in C_{(t, s)}(A)$ and so $\mu_{A}(a) \geq t, \gamma_{A}(a) \leq s$, i.e., $\langle a ;(t, s)\rangle \in A$. This shows that (q4) is valid. Let $a, b \in S, t_{1}, t_{2} \in(0,1]$ and $s_{1}, s_{2} \in[0,1)$ be such that $\left\langle a ;\left(t_{1}, s_{1}\right)\right\rangle \overline{\bar{\epsilon} \vee \overline{\mathrm{q}_{k}}} A$ and $\left\langle b ;\left(t_{2}, s_{2}\right)\right\rangle \overline{\bar{\epsilon} \vee \overline{\mathrm{q}_{k}}} A$. Then $\left\langle a ;\left(t_{1}, s_{1}\right)\right\rangle \in A,\left\langle a ;\left(t_{1}, s_{1}\right)\right\rangle \mathrm{q}_{k} A$ and $\left\langle b ;\left(t_{2}, s_{2}\right)\right\rangle \in A,\left\langle b ;\left(t_{2}, s_{2}\right)\right\rangle \mathrm{q}_{k} A$, which implies that $a \in C_{(t, s)}(A) \subseteq C_{\left(\min \left\{t_{1}, t_{2}\right\}, \max \left\{s_{1}, s_{2}\right\}\right)}(A)$ and $b \in C_{(t, s)}(A) \subseteq C_{\left(\min \left\{t_{1}, t_{2}\right\}, \max \left\{s_{1}, s_{2}\right\}\right)}(A)$. Since $C_{\left(\min \left\{t_{1}, t_{2}\right\}, \max \left\{s_{1}, s_{2}\right\}\right)}(A)$ is bi-ideal of $S$ by (e4), it follows by (b2) that $a b \in C_{\left(\min \left\{t_{1}, t_{2}\right\}, \max \left\{s_{1}, s_{2}\right\}\right)}(A)$, that is, $\mu_{A}(a b) \geq \min \left\{t_{1}, t_{2}\right\}$, and $\gamma_{A}(a b) \leq$ $\max \left\{s_{1}, s_{2}\right\}$, so that $\left\langle a b ; \min \left\{t_{1}, t_{2}\right\}, \max \left\{s_{1}, s_{2}\right\}\right\rangle \in A$. Hence (q5) is valid. Finally, let $a, b, c \in S$, $t_{1}, t_{2} \in(0,1]$ and $s_{1}, s_{2} \in[0,1)$ be such that $\left\langle a ;\left(t_{1}, s_{1}\right)\right\rangle \overline{\bar{\epsilon} \vee \overline{\mathrm{q}_{k}}} A$ and $\left\langle c ;\left(t_{2}, s_{2}\right)\right\rangle \overline{\bar{\epsilon} \vee \overline{\mathrm{q}_{k}}} A$. Then $\left\langle a ;\left(t_{1}, s_{1}\right)\right\rangle \in A,\left\langle a ;\left(t_{1}, s_{1}\right)\right\rangle \mathrm{q}_{k} A$ and $\left\langle c ;\left(t_{2}, s_{2}\right)\right\rangle \in A,\left\langle c ;\left(t_{2}, s_{2}\right)\right\rangle \mathrm{q}_{k} A$, which implies that $a \in C_{(t, s)}(A) \subseteq C_{\left(\min \left\{t_{1}, t_{2}\right\}, \max \left\{s_{1}, s_{2}\right\}\right)}(A)$ and $c \in C_{(t, s)}(A) \subseteq C_{\left(\min \left\{t_{1}, t_{2}\right\}, \max \left\{s_{1}, s_{2}\right\}\right)}(A)$. Since $C_{\left(\min \left\{t_{1}, t_{2}\right\}, \max \left\{s_{1}, s_{2}\right\}\right)}(A)$ is bi-ideal of $S$ by (e4), it follows by (b3) that $a b c \in$ $C_{\left(\min \left\{t_{1}, t_{2}\right\}, \max \left\{s_{1}, s_{2}\right\}\right)}(A)$, that is, $\mu_{A}(a b c) \geq \min \left\{t_{1}, t_{2}\right\}$, and $\gamma_{A}(a b c) \leq \max \left\{s_{1}, s_{2}\right\}$, so that $\left\langle a b c ; \min \left\{t_{1}, t_{2}\right\}, \max \left\{s_{1}, s_{2}\right\}\right\rangle \in A$. Hence (q6) is valid.

Therefore, as a concluding remark, we have the following theorem.

Theorem 3.17 For an IFS $A=\left\langle x, \mu_{A}, \gamma_{A}\right\rangle$ of $S$, the following are equivalent

(1) $A=\left\langle x, \mu_{A}, \gamma_{A}\right\rangle$ is an $\left(\bar{\epsilon}, \bar{\epsilon} \vee \overline{q_{k}}\right)$-intuitionistic fuzzy bi-ideal of $S$.

(2) $A=\left\langle x, \mu_{A}, \gamma_{A}\right\rangle$ satisfies the condition (e4).

(3) $A=\left\langle x, \mu_{A}, \gamma_{A}\right\rangle$ satisfies the three conditions (e1), (e2) and (e3).

For an IFS $A=\left\langle x ; \mu_{A}, \gamma_{A}\right\rangle$ of $S$, we consider the following sets

$$
\begin{aligned}
& \Gamma_{1}:=\left\{t \in(0,1] \mid U\left(\mu_{A} ; t\right) \neq \emptyset \Longrightarrow U\left(\mu_{A} ; t\right) \text { is a bi-ideal of } S\right\}, \\
& \Gamma_{2}:=\left\{s \in[0,1) \mid L\left(\mu_{A} ; t\right) \neq \emptyset \Longrightarrow L\left(\mu_{A} ; t\right) \text { is a bi-ideal of } S\right\} .
\end{aligned}
$$

Then

(1) If $\Gamma_{1}=(0,1]$ and $\Gamma_{2}=[0,1)$, then $A=\left\langle x ; \mu_{A}, \gamma_{A}\right\rangle$ is an intuitionistic fuzzy bi-ideal of $S$.

(2) If $\Gamma_{1}=(0,0.5]$ and $\Gamma_{2}=[0.5,1)$, then $A=\left\langle x ; \mu_{A}, \gamma_{A}\right\rangle$ is an $(\in, \in \vee \mathrm{q})$-intuitionistic fuzzy bi-ideal of $S$. 
(3) If $\Gamma_{1}=\left(0, \frac{1-k}{2}\right]$ and $\Gamma_{2}=\left[\frac{1-k}{2}, 1\right)$, then $A=\left\langle x ; \mu_{A}, \gamma_{A}\right\rangle$ is an $\left(\in, \in \vee \mathrm{q}_{k}\right)$-intuitionistic fuzzy bi-ideal of $S$.

(4) If $\Gamma_{1}=\left(\frac{1-k}{2}, 1\right]$ and $\Gamma_{2}=\left[0, \frac{1-k}{2}\right)$, then $A=\left\langle x ; \mu_{A}, \gamma_{A}\right\rangle$ is an $\left(\bar{\epsilon}, \bar{\epsilon} \vee \overline{q_{k}}\right)$-intuitionistic fuzzy bi-ideal of $S$.

\section{Competing interests}

The authors declare that they have no competing interests.

\section{Authors' contributions}

AK proposed the structure, reviewed the mathematical concepts and coordinated the manuscript. BD participated in its design and helped to check the examples constructed for new concepts in the manuscript. NHS linguistically edited, sequenced and drafted the manuscript. HK introduced new concepts, proofs to mathematical results and typed the manuscript.

\section{Author details}

${ }^{1}$ Department of Mathematics, Abdul Wali Khan University, Mardan, Khyber Pakhtoon Khwa, Pakistan. ${ }^{2}$ Department of Mathematics, Yazd University, Yazd, Iran. ${ }^{3}$ Department of Mathematical Sciences, Faculty of Science, Universiti Teknologi Malaysia, UTM Johor Bahru, Johor, 81310, Malaysia.

Received: 7 March 2013 Accepted: 31 July 2013 Published: 21 August 2013

\section{References}

1. Kehayopulu, N, Tsingelis, M: Fuzzy bi-ideals in ordered semigroups. Inf. Sci. 171, 13-28 (2005)

2. Mordeson, JN, Malik, DS, Kuroki, N: Fuzzy Semigroups. Studies in Fuzziness and Soft Computing, vol. 131. Springer, Berlin (2003)

3. Murali, V: Fuzzy points of equivalent fuzzy subsets. Inf. Sci. 158, 277-288 (2004)

4. Bhakat, SK, Das, P: $(\in, \in \vee$ q)-fuzzy subgroups. Fuzzy Sets Syst. 80, 359-368 (1996)

5. Bhakat, SK, Das, P: Fuzzy subrings and ideals redefined. Fuzzy Sets Syst. 81, 383-393 (1996)

6. Davvaz, B: Fuzzy R-subgroups with thresholds of near-rings and implication operators. Soft Comput. 12, 875-879 (2008)

7. Jun, YB, Khan, A, Shabir, M: Ordered semigroups characterized by their $(\in, \in \vee q)$-fuzzy bi-ideals. Bull. Malays. Math. Soc. 32(3), 391-408 (2009)

8. Davvaz, B, Khan, A: Characterizations of regular ordered semigroups in terms of $(\alpha, \beta)$-fuzzy generalized bi-ideals. Inf. Sci. 181, 1759-1770 (2011)

9. Shabir, M, Jun, YB, Nawaz, Y: Characterization of regular semigroups by $(\alpha, \beta)$-fuzzy ideals. Comput. Math. Appl. 59, 161-175 (2010)

10. Jun, YB: Generalizations of $(\epsilon, \in \vee q)$-fuzzy subalgebras in BCK/BCl-algebras. Comput. Math. Appl. 58, 1383-1390 (2009)

11. Jun, YB, Khan, A, Sarmin, NH, Khan, H: Bi-ideals of ordered semigroups based on the intuitionistic fuzzy points (submitted)

doi:10.1186/1029-242X-2013-397

Cite this article as: Khan et al.: Redefined intuitionistic fuzzy bi-ideals of ordered semigroups. Journal of Inequalities and Applications 2013 2013:397.

\section{Submit your manuscript to a SpringerOpen ${ }^{\circ}$ journal and benefit from:}

- Convenient online submission

- Rigorous peer review

Immediate publication on acceptance

Open access: articles freely available online

- High visibility within the field

- Retaining the copyright to your article 